\title{
Quelques engins de pêche en eau douce d'Afrique centrale
}

A catalogue of freshwater fishing implements from Central Africa

\section{Serge Bahuchet et Pauline Rameau}

\section{(2) OpenEdition}

Journals

Édition électronique

URL : http://journals.openedition.org/ethnoecologie/2877

DOI : 10.4000/ethnoecologie.2877

ISSN : 2267-2419

Éditeur

Laboratoire Eco-anthropologie et Ethnobiologie

Référence électronique

Serge Bahuchet et Pauline Rameau, "Quelques engins de pêche en eau douce d'Afrique centrale », Revue d'ethnoécologie [En ligne], 10 | 2016, mis en ligne le 31 décembre 2016, consulté le 19 avril 2019. URL : http://journals.openedition.org/ethnoecologie/2877 ; DOI : 10.4000/ethnoecologie.2877

Ce document a été généré automatiquement le 19 avril 2019

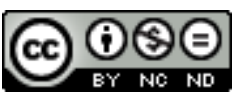

Revue d'ethnoécologie est mis à disposition selon les termes de la licence Creative Commons Attribution - Pas d'Utilisation Commerciale - Pas de Modification 4.0 International. 


\section{Quelques engins de pêche en eau douce d'Afrique centrale}

A catalogue of freshwater fishing implements from Central Africa

Serge Bahuchet et Pauline Rameau

\section{Les engins de pêche}

1 Les programmes collectifs menés en Afrique centrale (essentiellement République centrafricaine, Cameroun, Gabon) sur l'ethnoécologie et sur l'alimentation des communautés forestières (Bahuchet et al. 2001, Froment et al. 1996, Hladik et al. 1989), ainsi que des missions ou des recherches individuelles (telles que Sabinot 2008, Soengas 2010), ont été accompagnés de collectes d'objets, dont une petite partie concerne les activités de pêche.

2 Nous conservons ainsi une trentaine d'objets, des cinq pays d'Afrique centrale de l'Ouest (Cameroun, 7 ; Congo, 3 ; Gabon, 14 ; RCA, 4 ; RDC, 1), recueillis par huit chercheurs entre 1970 et 2015. Ce sont des hameçons, des pointes de harpons, des nasses, des écopes et divers paniers.

3 L'article de Dounias, Cogels, Mvé Mbida \& Carrière (2016) décrit et illustre plusieurs de ces techniques, dans lesquelles des engins similaires à ceux de nos collections sont employés. 


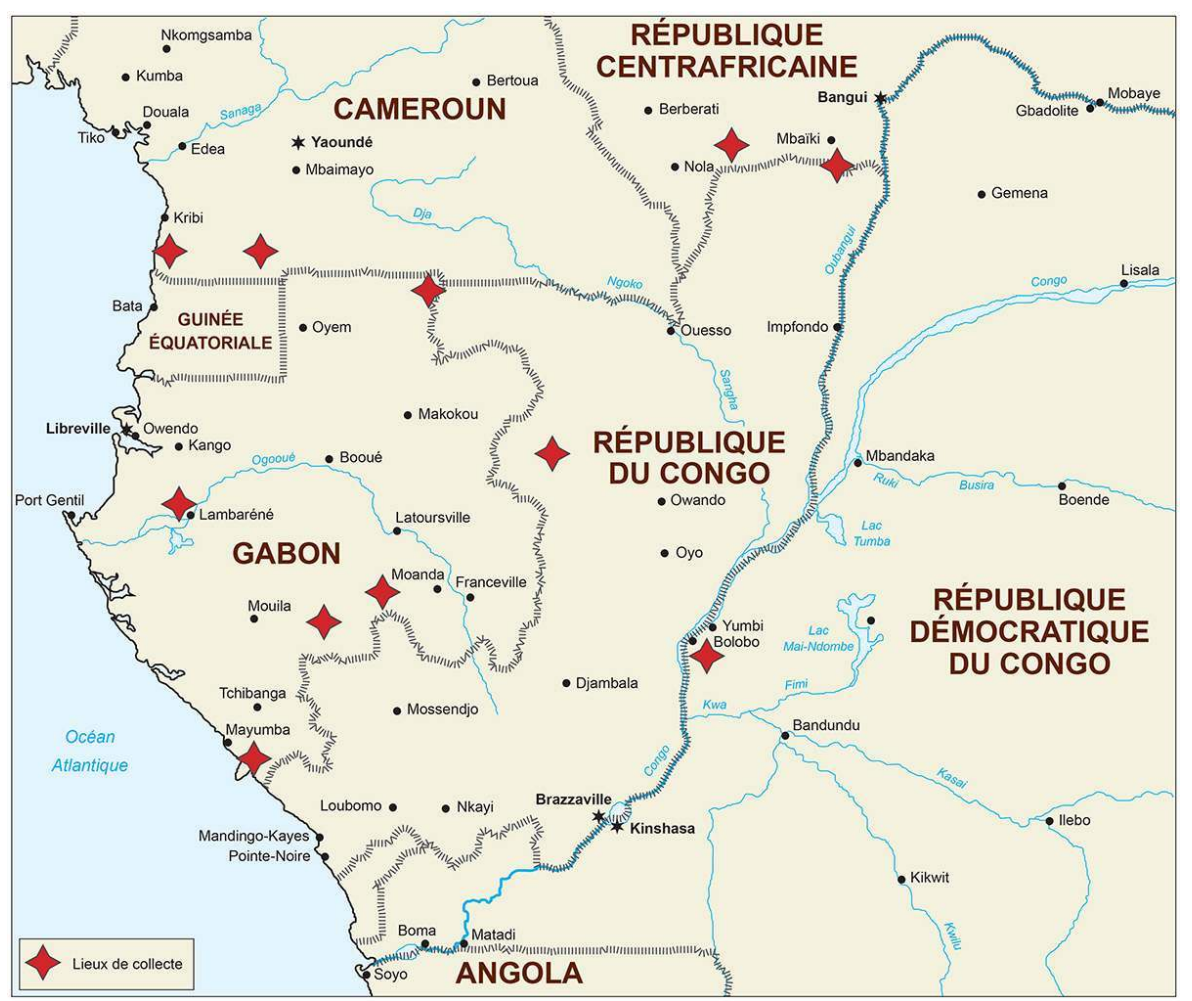

(c) L. Venot

\section{La difficile classification des engins de pêche}

La pêche est une activité universelle qui mobilise beaucoup de types d'engins différents, et il en existe une très grande diversité tant à l'échelle mondiale que localement. Pour s'y retrouver, de nombreuses classifications et des terminologies complexes ont été réalisées ; la plus utilisée est celle de la FAO qui est, comme la plupart, basées sur la forme de l'ustensile et son efficacité technique (Nédélec 1982).

En étudiant l'ensemble des techniques humaines d'action sur la matière, Leroi-Gourhan (1973) avait proposé un point de vue différent, plus englobant, en montrant comment les techniques de capture ne différaient pas dans leur principe technique, mécaniquement, sinon par la proie recherchée. Il démontrait ainsi qu'il n'y a pas de spécificité de la pêche par rapport à la chasse et à la guerre, ce sont les mêmes principes physiques et les mêmes armes et techniques qui sont en jeu.

Cependant un système de classification se distingue, qui est le seul à prendre en compte la diversité des rapports entre l'homme et l'animal. C'est celui proposé par Théodore Monod (1973), fondé sur « la nature de l'action (principale) subie par le poisson ». Il accorde une importance primordiale à l'action de l'outil de capture, au «type de menace » qu'il représente pour l'animal, rejetant au second plan forme et nature de l'objet. Monod appelle joliment cela « le point de vue du poisson ». En effet il classe les techniques et les engins en fonction de leur place par rapport au corps $d u$ poisson et leur action sur celui. De ce point de vue cette classification est réellement ethnobiologique. 
7 Monod distingue 12 classes, qui toutes ne mobilisent pas des outils; ainsi les techniques de pincement qui saisissent le poisson (à la main, avec le bec du Cormoran...), de percussion où on l'assomme, celles où on en perfore le corps en projetant l'arme (arc et flèches, harpons, foënes) ou bien par un hameçon, ou encore celles où le corps du poisson est contraint par des parois immobilisantes, qu'elles soient actives (épervier, carrelets, chaluts...) ou passives (filets maillants, nasses, casiers...), sans oublier les techniques paralysantes (poisons de pêche, électricité) et celles où l'on ramasse les poissons par assèchement (écopage)...

8 Notre collection d'engins d'Afrique centrale ne couvre qu'une partie de ces catégories, mais elle est bien caractéristique des principales techniques employées dans les eaux continentales de la forêt africaine. Prédominent en effet les engins «à parois immobilisantes ", c'est-à-dire les nasses et paniers de capture.

9 C'est donc en suivant la classification fonctionnelle de Monod que nous établirons notre catalogue.

\section{Catalogue des engins de pêche selon la « classification fonctionnelle » de Théodore Monod}

10 Chaque objet est présenté sous forme d'une fiche, accompagnée d'une photographie, comportant les éléments suivants :

- Nom de l'objet

- Nom de l'ethnie. Localisation: Pays, village, région (département)

- Description : usage, matières.

- Date de collecte. Nom du collecteur.

- Dimensions : hauteur x longueur x largeur, en centimètres.

- Numéro d'inventaire dans la collection d'ethnobiologie (ETB-code pays-collecteurannée-nºbjet).

\section{Engins agissant par perforation (Classe IV)}

Perforation balistique. Engins barbelés

11 Le pêcheur projette l'arme vers sa proie.

1 - Harpon emmanché 
Figure 2 : Harpon emmanché mbete - ETB-CG-AE-2003-003

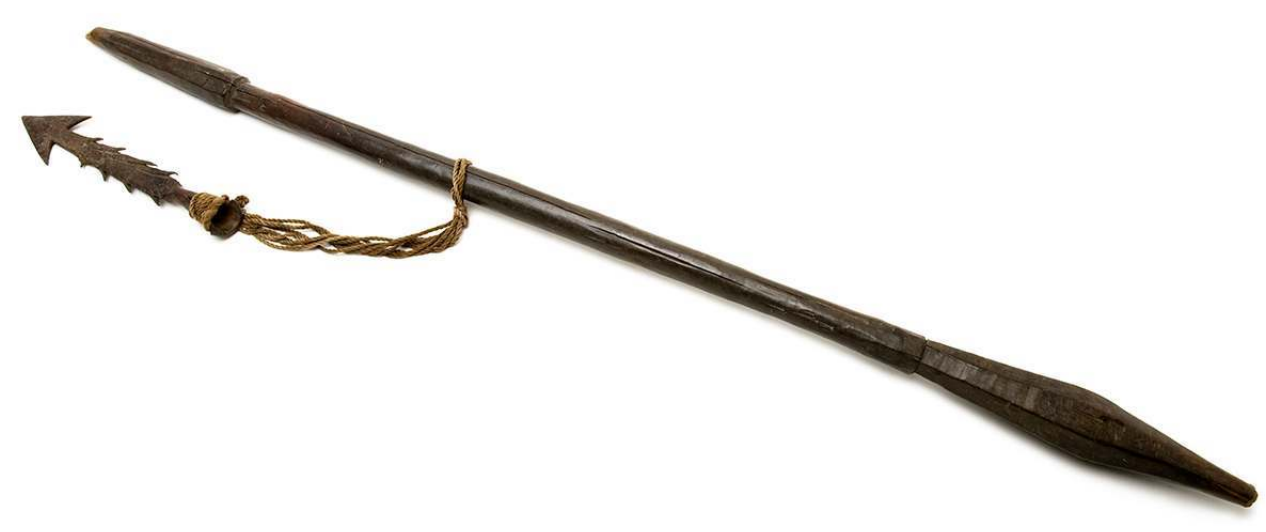

\section{(c) J.C. Domenech/MNHN}

12 - Mbete. Congo. Mbomo, Région de Kelle (Cuvette ouest)

- Pour la chasse aux hippopotames. Pointe de fer détachable, retenue au manche de bois par une ligature tressée en écorce de Manniophyton fulvum (Euphorbiacée).

- Juin 2003 ; collecteur Alain Epelboin

- Dimensions : hampe : $104 \times$ x 5,5; pointe : 24 x 5,5

- $\mathrm{N}^{\circ}$ d'inventaire : ETB-CG-AE-2003-003

\section{2 - Fers de harpon}

Figure 3 : Deux pointes de harpons mbete ETB-CG-AE-2003-001 et ETB-CG-AE-2003-002

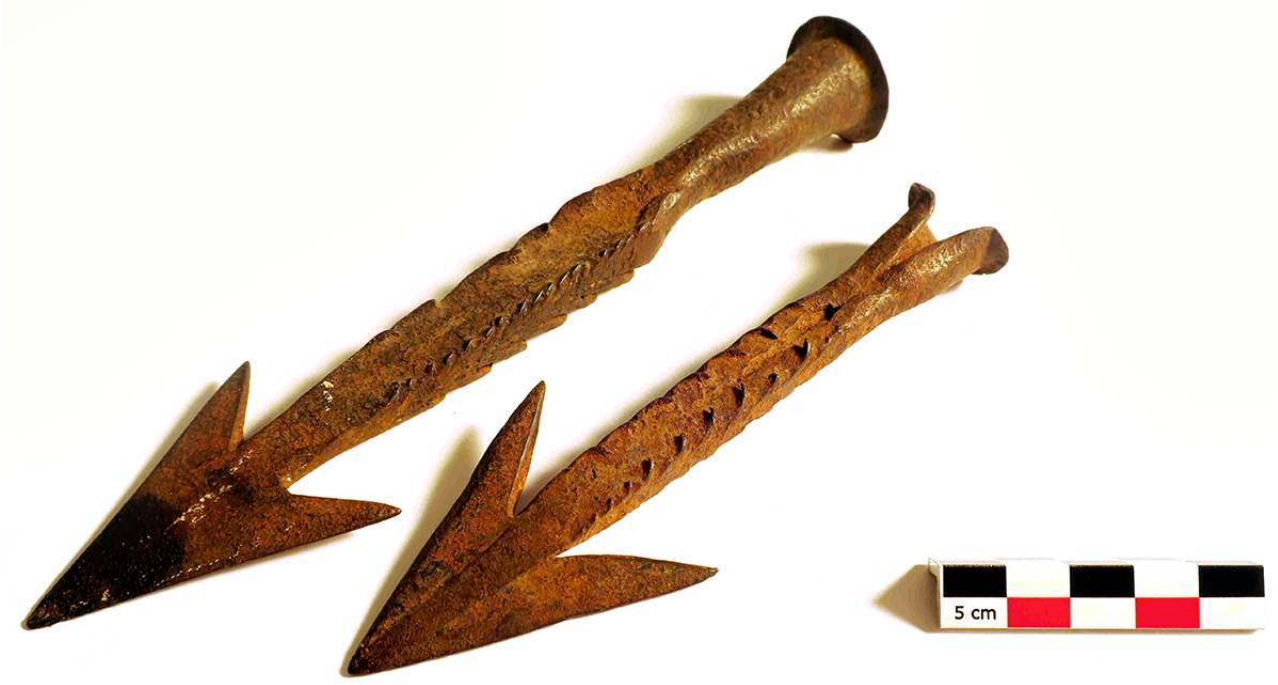

(C) P. Rameau 
13 - Mbete. Congo. Mbomo, Région de Kelle (Cuvette ouest)

- Pour la chasse aux hippopotames.

- Juin 2003 ; collecteur Alain Epelboin

- Dimensions : 15,5 x $5 ; 21$ x 5,5

- $\mathrm{N}^{\circ}$ d'inventaire : ETB-CG-AE-2003-001 ; ETB-CG-AE-2003-002

\section{3 - Fers de harpon}

Figure 4 : Deux pointes de harpons akele ETB-GA-AP-2012-001 et ETB-GA-AP-2012-002

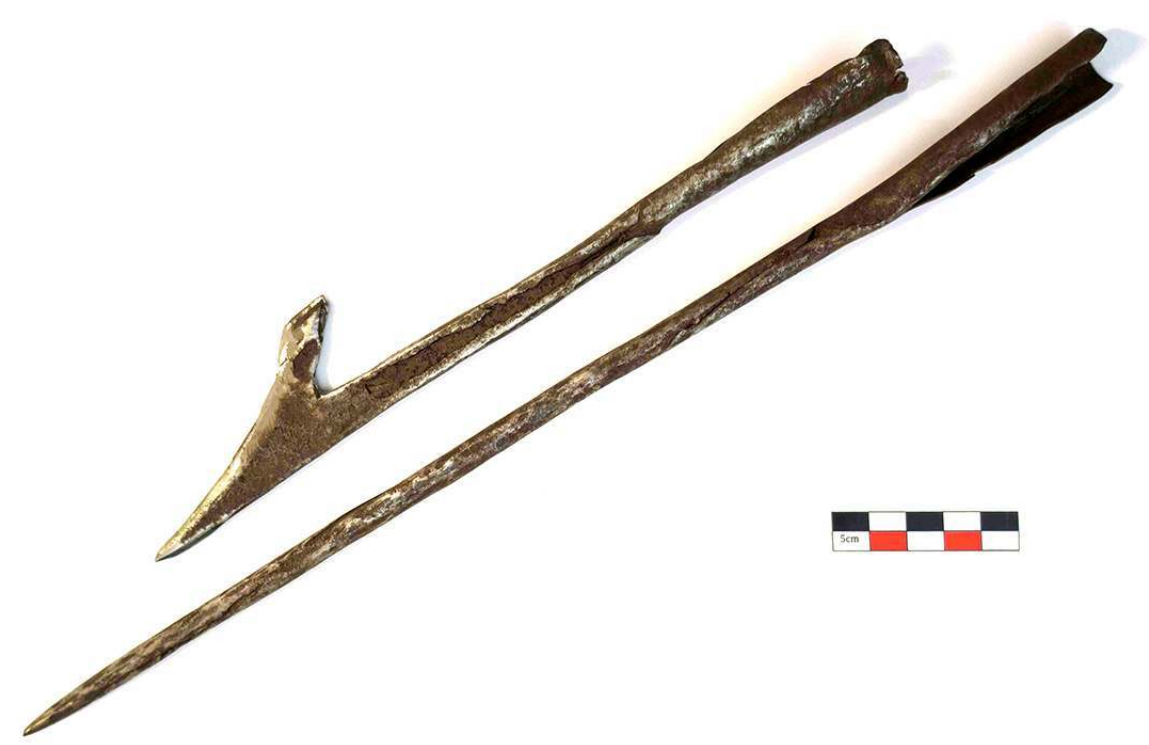

(c) P. RAMEAU

14 - Akele (Ongom). Gabon. Makouké, Région de Lambaréné (Moyen Ogooué)

- Pointe droite (français local «sagaie »). Pour la pêche des gros poissons dans l'Ogooué, dans les roselières. Pointe à ergot (français local « harpon»), projetée pour la pêche à partir de la pirogue. Forgé à froid, à partir d'une lame de pelle découpée au burin et martelée à la masse.

- 15 décembre 2012 ; collectrice Adèle de Palaminy

- Dimensions : pointe droite, longueur 34,5; pointe à ergot, 24,5 x 3,5

- $\mathrm{N}^{\circ}$ d'inventaire: pointe droite: ETB-GA-AP-2012-001; pointe à ergot ETB-GAAP-2012-002

\section{Perforation non balistique. Action localisée (buccale)}

15 L'élément perforant est suspendu à un support (en général une ligne), et la proie s'y accroche par la bouche en mordant un appât.

\section{4 - Ensemble de trois hameçons}




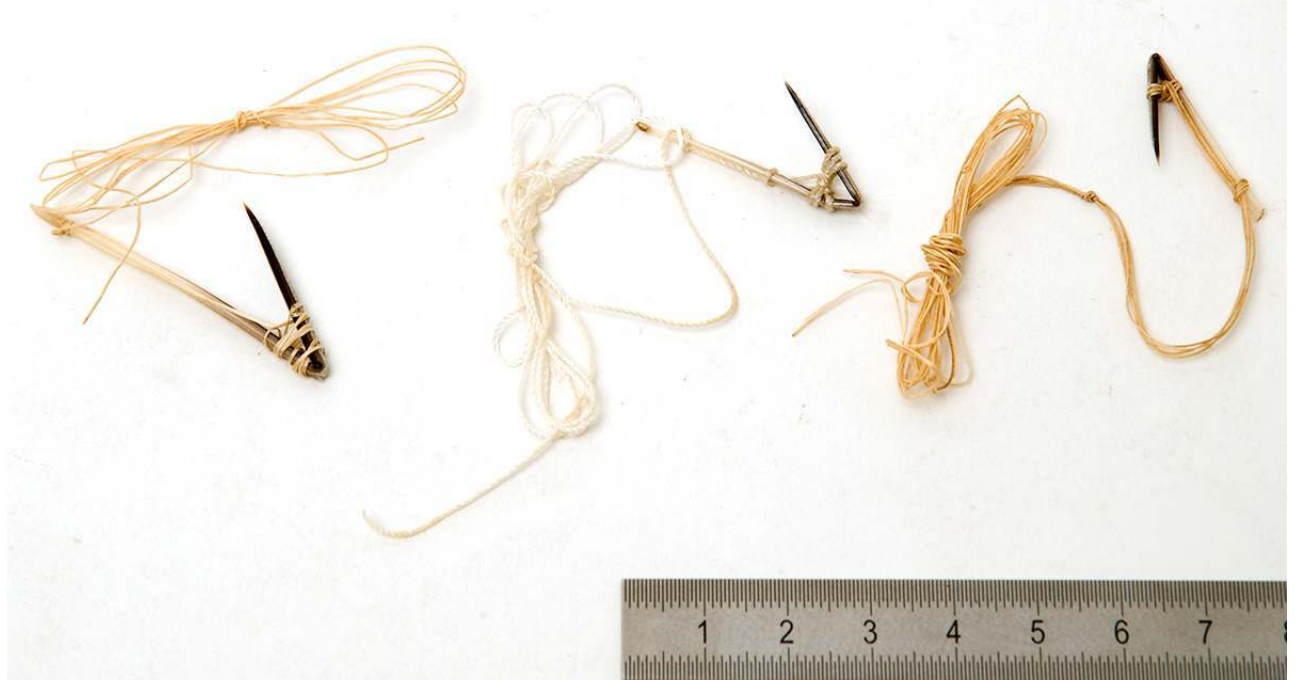

\section{() J.C. DOMENECH/MNHN}

- Ntumu, Cameroun, village de Nkong Meyos (Ntem)

- Destinés plus particulièrement aux silures, avec appât. Piquants d'Athérure (Atherurus africanus) repliés, liés par un fil de racine aérienne d'Aracée épiphyte Cercestis mirabilis (= Rhektophyllum mirabile). Hameçons fabriqués par un Ntumu originaire de Guinée Équatoriale.

- Dimensions : 4,5 x 1,5; 3 × 1, 5 et 2,5 $\times 1$

- Décembre 1994 ; collecteur Serge Bahuchet

- $\mathrm{N}^{\circ}$ d'inventaire : ETB-CM-SB-2016-003a, 003b, 003c

\section{Engins à parois immobilisantes (Cl. VIII)}

\section{Engin actif, actionné en poussant ou levant}

Dans cette famille, le ou les pêcheurs manipulent l'engin, pour intercepter le poisson.

Monod ne décrit pas spécifiquement la technique collective d'Afrique centrale, où un ensemble de pêcheurs avançant en ligne relève régulièrement à la main des filets sur cadre ou des paniers tenus face à soi.

\section{5 - Panier ovale}




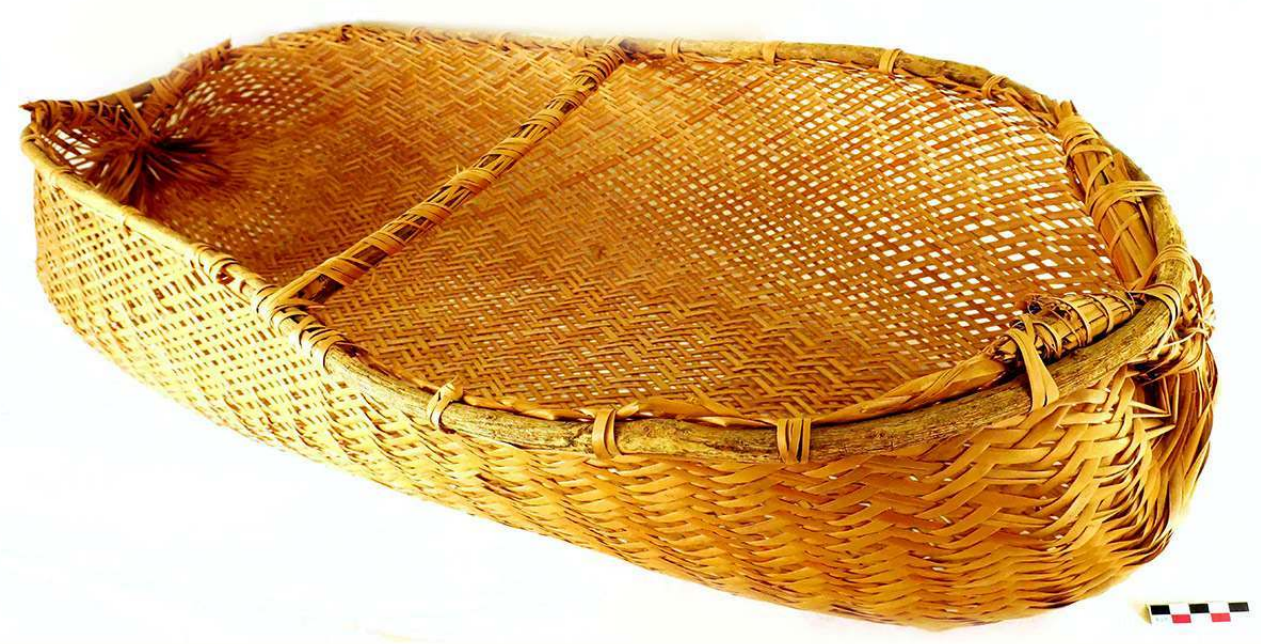

(c) P. Rameau

19 - Pande, RCA, Ngoto (Lobaye)

- Les femmes tiennent ces paniers à la main, devant elles, avançant en ligne côtes à côtes dans les cours d'eau. Elles plongent et relèvent leur panier tout en avançant. Matières : tiges de Marantacées, armature de bois

- 1979 ; collecteur Serge Bahuchet

- Dimensions : 2,5 x 102,5 x 42,5

- $\mathrm{N}^{\circ}$ d'inventaire : ETB-CF-SB-2016-001

\section{6 - Trouble, filet sur cadre}




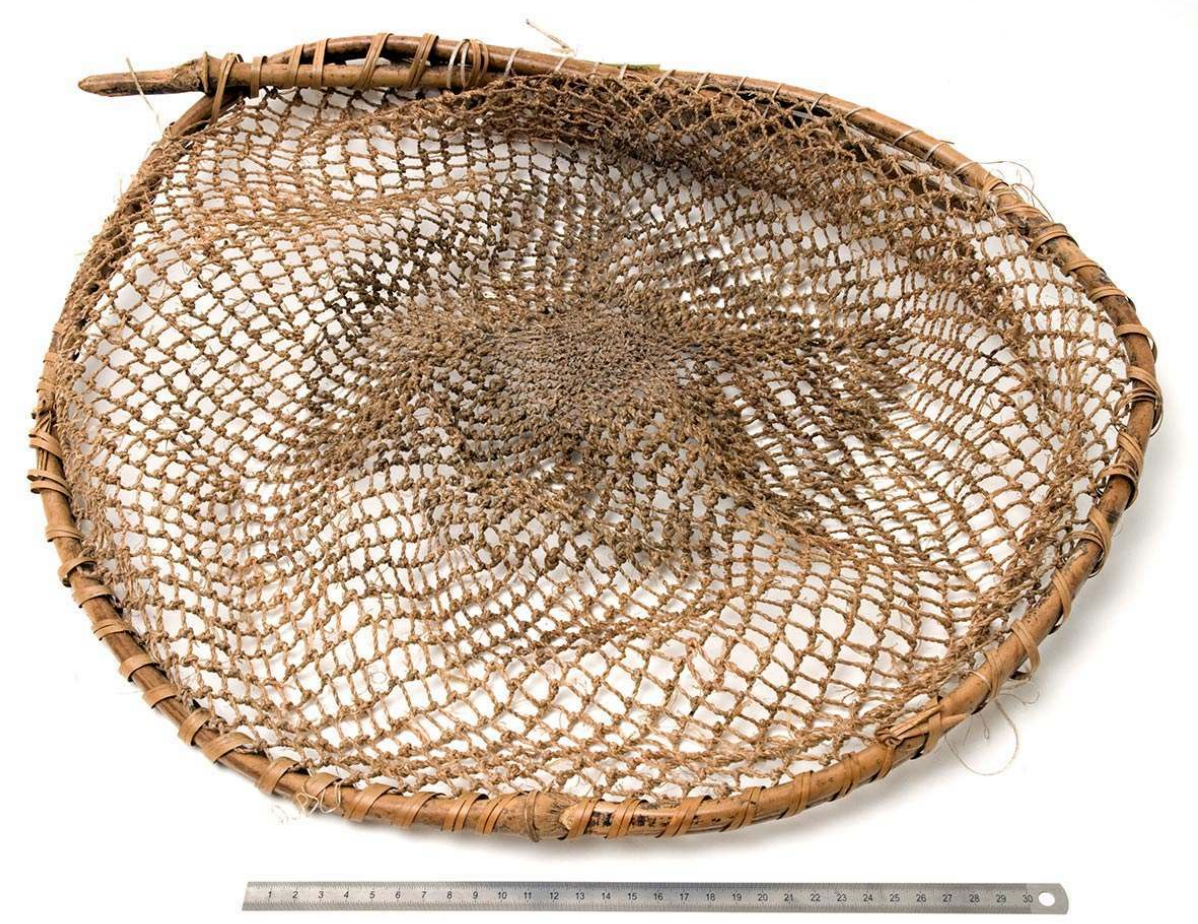

\section{(C) J.C. DOMENECH/MNHN}

- Ntumu, Cameroun, Nkong Meyos (Océan)

- Destiné aux crevettes et aux petits poissons. En fils de nylon extraits des sacs de farine ou de riz. Cadre: Hypselodelphys scandens (Marantacée). Ces filets-troubles à tout faire, employés généralement en groupe, servent aussi bien à des pêches collectives, en avançant en ligne, qu'à fouiller l'eau stagnante lors des parties d'écopage (voir plus bas).

- Décembre 1994 ; collecteur Serge Bahuchet

- Dimensions : profondeur de la poche 16 × 53,5 x 46

- $\mathrm{N}^{\circ}$ d'inventaire : ETB-CM-SB-1994-002 
Figure 8: Groupes de femmes ntumu partant pour la pêche collective. Nkong Meyos, décembre 1994

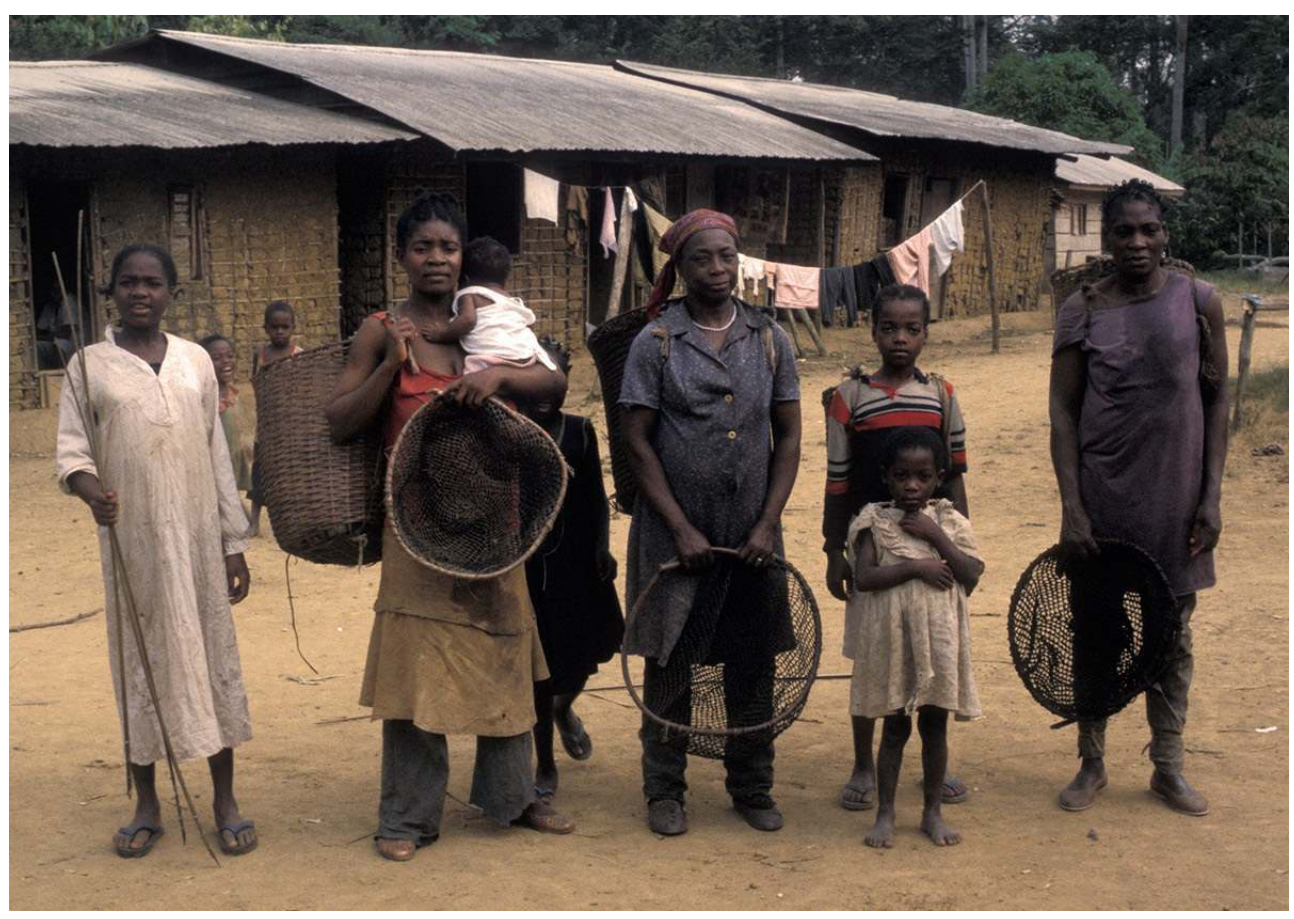

(c) S. Bahuchet

\section{7 - Matériel annexe : deux paniers de ceinture}

Pendant les parties de pêche, les femmes portent à la ceinture de petits paniers dans lesquels elles placent leurs prises au fur et à mesure, avant de verser l'ensemble dans les hottes, plus grandes, restées sur le bord. 


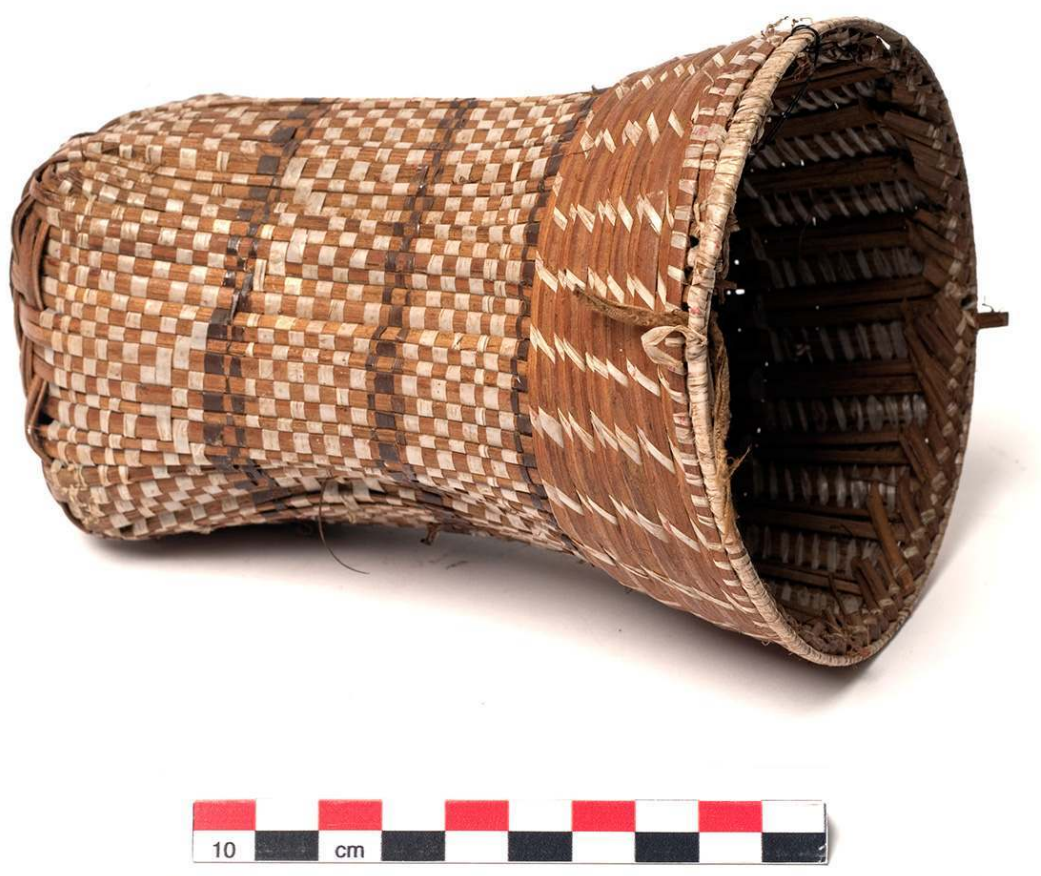

(c) J.C. Domenech/MNH

22 - Akele, Gabon, village Dienga (Ngounié)

- En lamelles de Marantacées avec des fils de plastique. Ce petit panier de femme se porte à la ceinture pendant les pêches à l'écope, pour y placer les petits poissons ramassés

- 28 avril 2011 ; collecteurs Serge Bahuchet, Marine Robillard et Sylvie Le Bomin

- Dimensions : 7 × 7 × 16,5

- No d'inventaire : ETB-GA-SB/MR-2011-014 


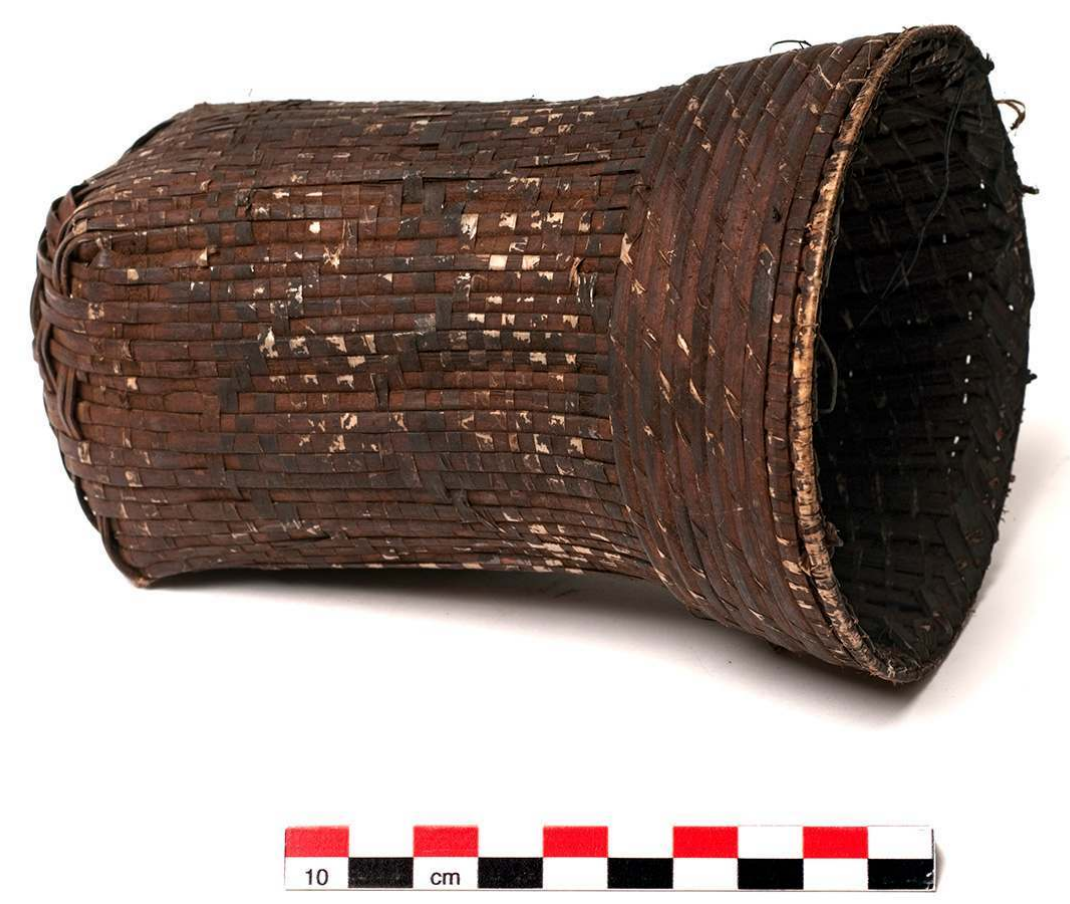

(c) J.C. Domenench/MNHN

23 - Akele, Gabon, village Dienga (Ngounié)

- En lamelles de Marantacées avec des fils de plastique. Ce petit panier de femme se porte à la ceinture pendant les pêches à l'écope, pour y placer les petits poissons ramassés

- 28 avril 2011 ; collecteurs Serge Bahuchet, Marine Robillard et Sylvie Le Bomin

- Dimensions : 7 × 7 × 15,5

- $\mathrm{N}^{\circ}$ d'inventaire : ETB-GA-SB/MR-2011-015

\section{Engin passif, agissant par emprisonnement}

24 Dans cette famille, l'engin est fixe, et les proies se prennent à l'intérieur, soit par leur propre mouvement soit poussées par le courant.

\section{Avec mécanisme}

8 - Piège à crabe 


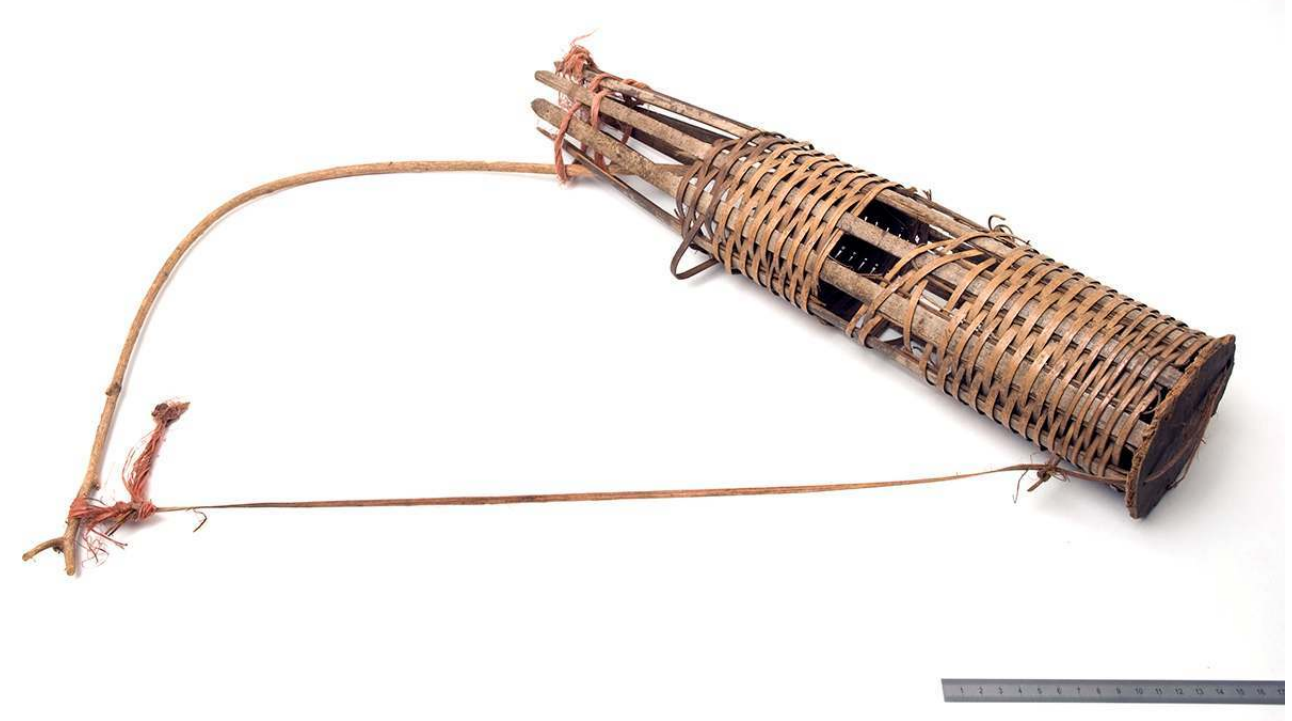

(C) J.C. DOMENECH/MNHN

- Yasa ; Cameroun ; Ebodié (Océan)

- Piège-cage à fermeture mécanique, par ressort; fonctionne avec un appât (noix de palme). Destiné aux crabes de mangroves Cardisoma armatum. Se dispose à l'orifice du terrier des crabes. Cagette en lamelles de rotin et de raphia (armature) : tendeur en filament de rotin. Clapet en rondelle de bois compensé.

- 1984 ; collecteur Serge Bahuchet

- Dimensions : cagette : $52,5 \times 11$; arc, rayon 36 ; tendeur 60

- $\mathrm{N}^{\circ}$ d'inventaire : ETB-CM-SB-2016-005 
Figure 12: Croquis du piège à crabes

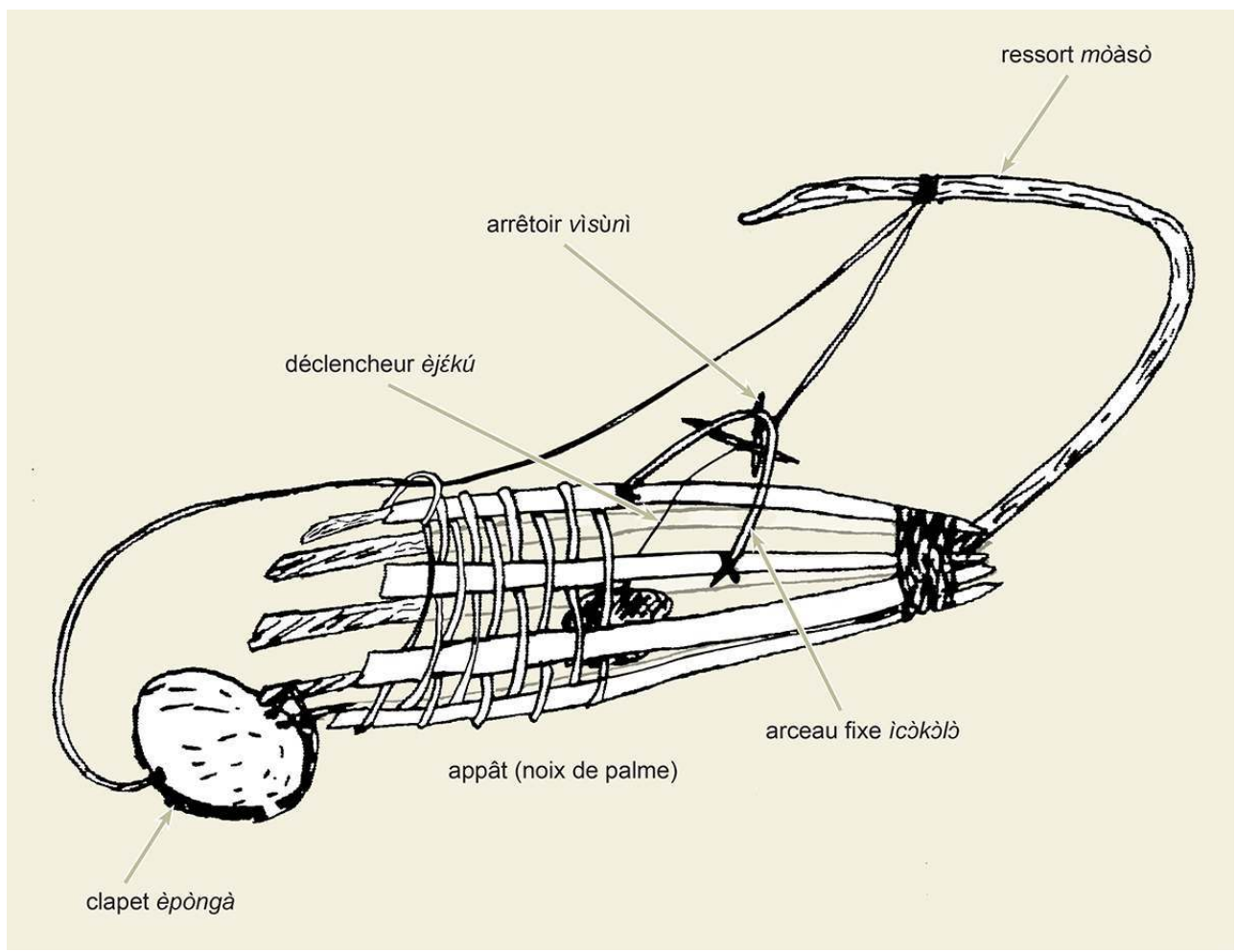

(In Bahuchet 1992)

Figure 13 : Les pièges et les crabes au retour de la pêche, Ebodié, 1984

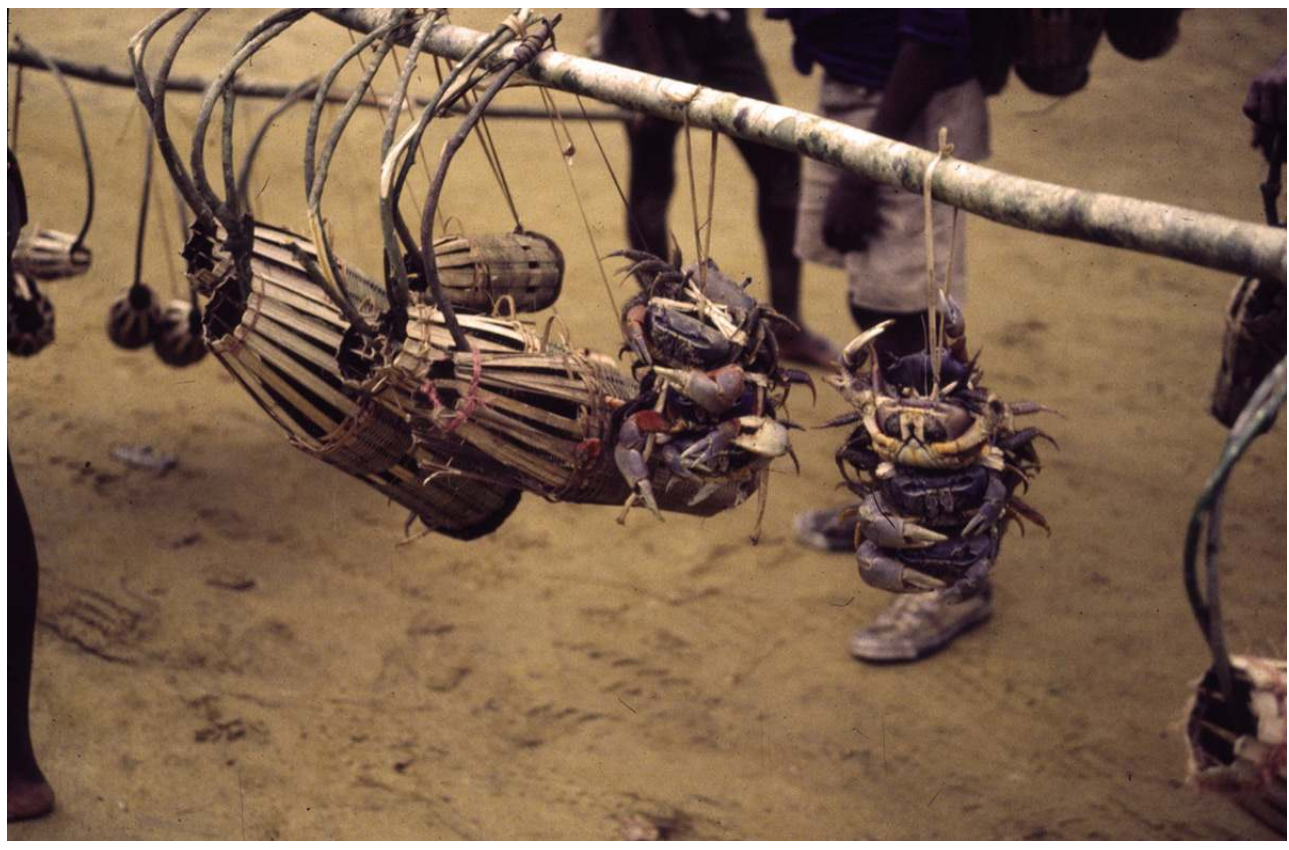

(c) S. Bahuchet 


\section{Avec un dispositif d'entrée}

Il s'agit de contenants où un goulet ou un entonnoir, fixe, empêche la proie de ressortir après qu'elle soit entrée, en général attirée par un appât. Cela regroupe la grande famille des nasses.

\section{9 - Nasse}

Figure 14 : Grande nasse dormante teke ETB-CD-VN-2013-001

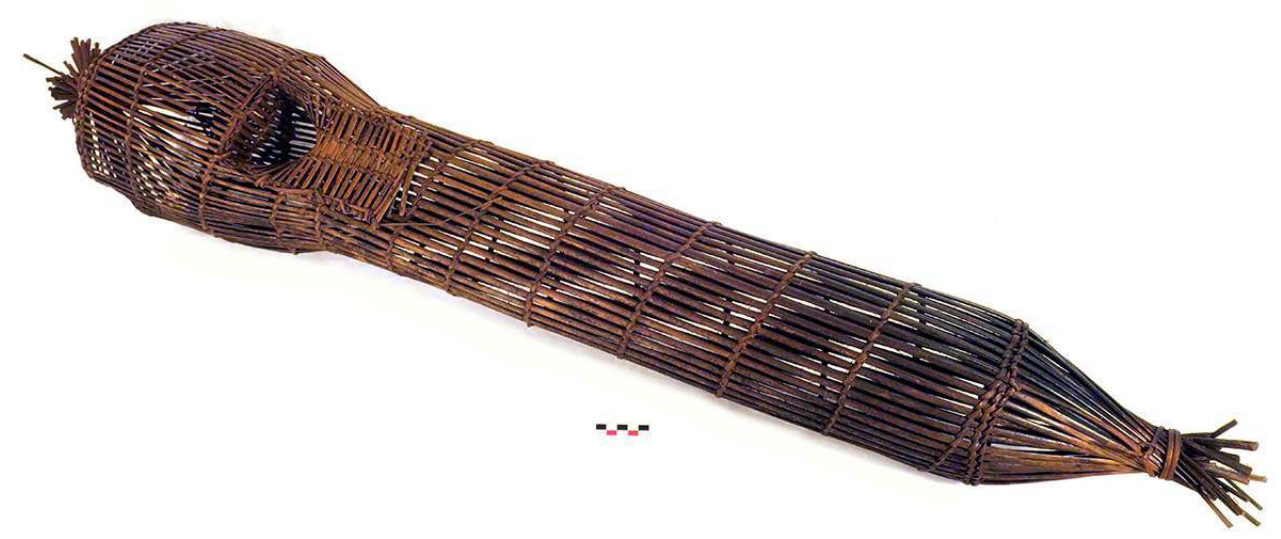

(C) P. Rameau

27 - Teke. République démocratique du Congo RDC. Embirima, Territoire de Bolobo (MaiNdombe)

- Grande nasse dormante, en lamelles de rotin. S'utilise au moment des variations de niveaux d'eau dans les petites rivières de forêt (transition saisons sèches/saison des pluies)

- Novembre 2013. Collecteurs Victor Narat, Flora Pennec et Sabrina Krief

- Dimensions : $127 \mathrm{x}$ diamètres 17/27

- $\mathrm{N}^{\circ}$ d'inventaire : ETB-CD-VN-2013-001

10 - Nasse

Figure 15 : Nasse akele ETB-GA-SB/MR-2011-043

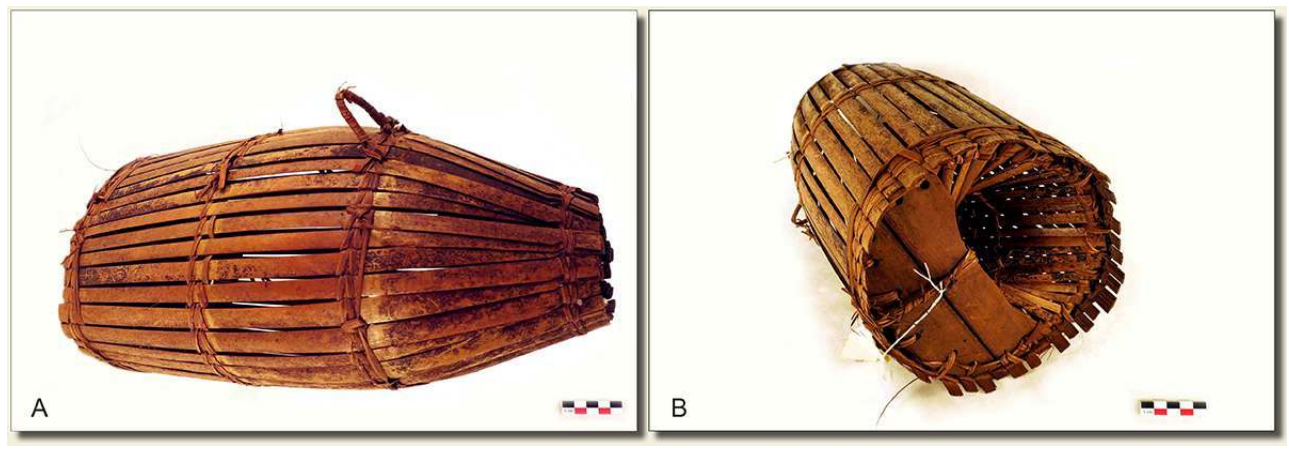

(c) P. Rameau 
- Akele, Gabon. Village Dienga (Ngounié)

- En écorces de palmes de raphia («bambou» en français local); plaquettes de bois à l'ouverture. Pour poissons, crabes et crevettes. De telles nasses peuvent être indifféremment disposées isolément ou en série dans des barrages

- Avril 2011 ; collecteurs Serge Bahuchet, Marine Robillard et Sylvie Le Bomin

- Dimensions : diamètre d'extrémité $9 \mathrm{x}$ gros diamètre $20 \mathrm{x}$ longueur 49

- $\mathrm{N}^{\circ}$ d'inventaire : ETB-GA-SB/MR-2011-043

Figure 16: Les nasses suspendues dans la cuisine. Dienga (Gabon), avril 2011

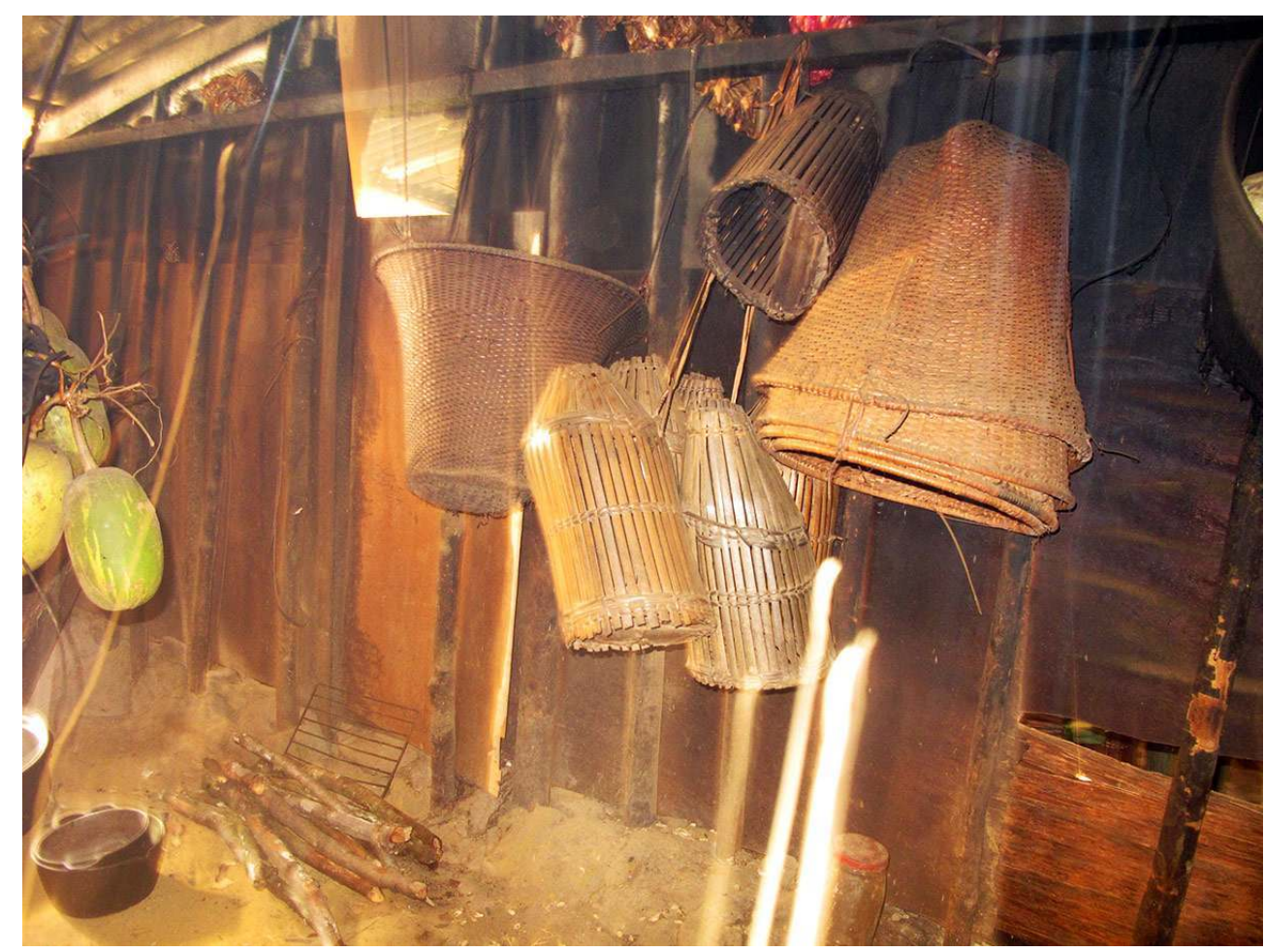

(c) S. Bahuchet

11 - Nasse

Figure 17 : Nasse bongo ETB-GA-SLB-2015-003

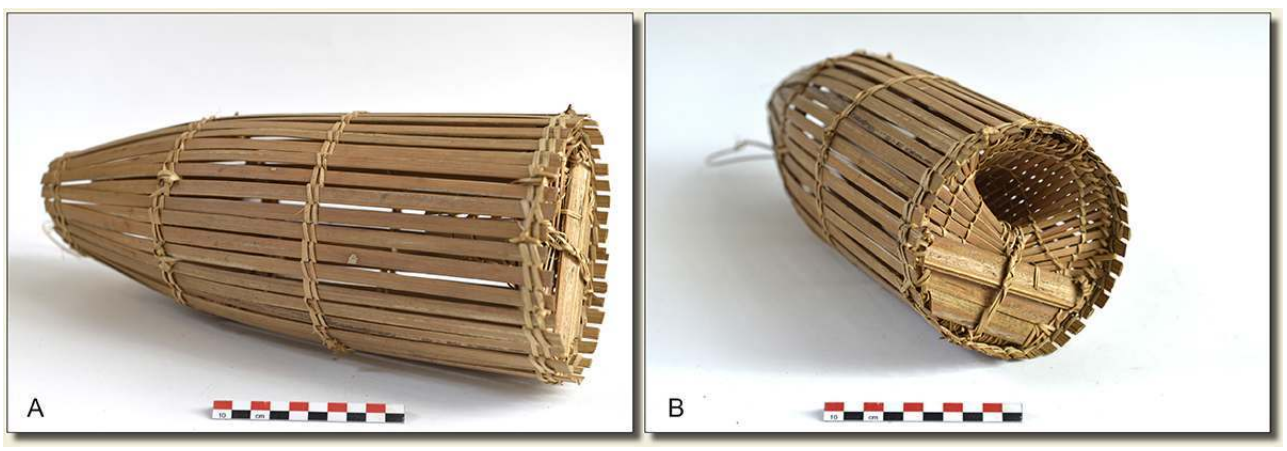

(c) J.C. Domenech/MNHN 
- Bongo, Gabon. Mouyikou (Ngounié)

- En écorce de palmes de raphia. Pour poissons, crabes et crevettes. De telles nasses peuvent être indifféremment disposées isolément ou en série dans des barrages

- Mars 2015 ; collectrice Sylvie Le Bomin

- Dimensions : 35 x 14

- $\mathrm{N}^{\circ}$ d'inventaire : ETB-GA-SLB-2015-003

- Cette nasse est exposée en permanence dans la Galerie de l'Homme.

12 - Nasse

Figure 18 A et B : Nasse lumbu ETB-GA-CS-2005-001

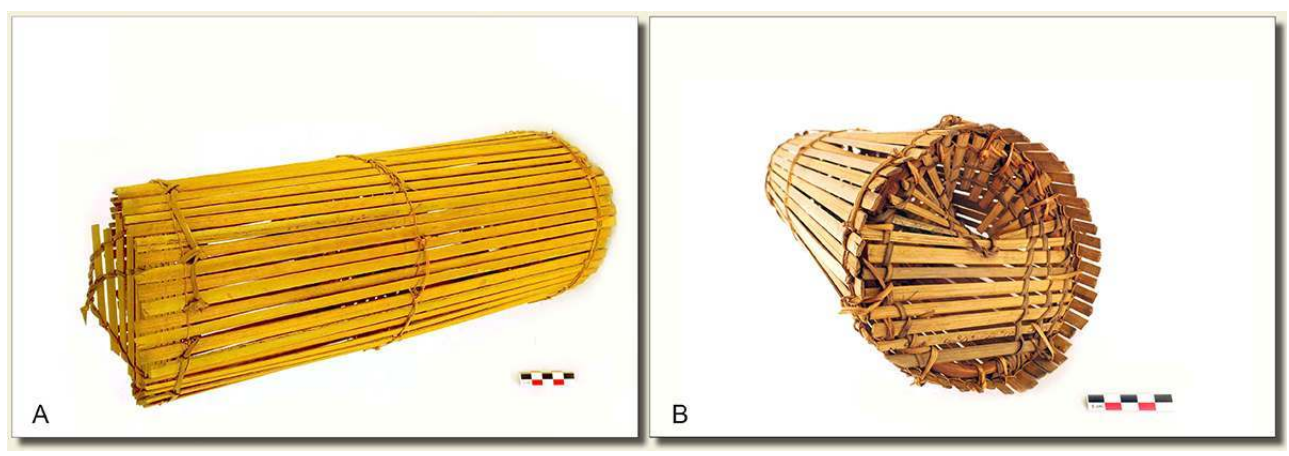

Cl. P. Rameau

30 - Lumbu, vili. Gabon. Socoma, Lagune Banio (Nyanga)

- Cylindriques; en éclisses de bambou de Chine. Pour crevettes et poissons chats. Avec appâts, en bordure des cours d'eau. Pêche réalisée par les femmes. (cf. Sabinot 2008)

- 2005 ; collectrice Catherine Sabinot

- Dimensions : 45 x $18: 19,5$

- $\mathrm{N}^{\circ}$ d'inventaire : Nasse cylindrique ; ETB-GA-CS-2005-001

13 - Matériel annexe : deux viviers 
Figure 19 : Vivier lumbu ETB-GA-CS-2005-003

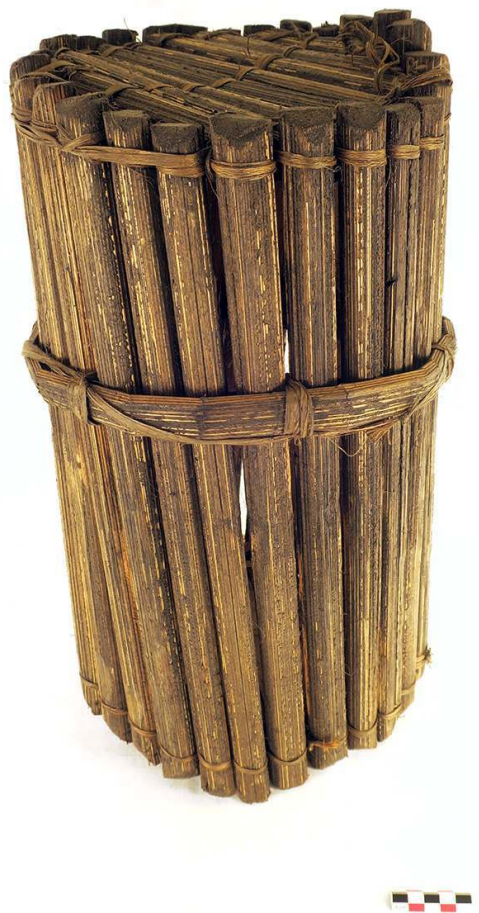

Cl. P. Rameau

Figure 20 : Vivier lumbu ETB-GA-CS-2005-002

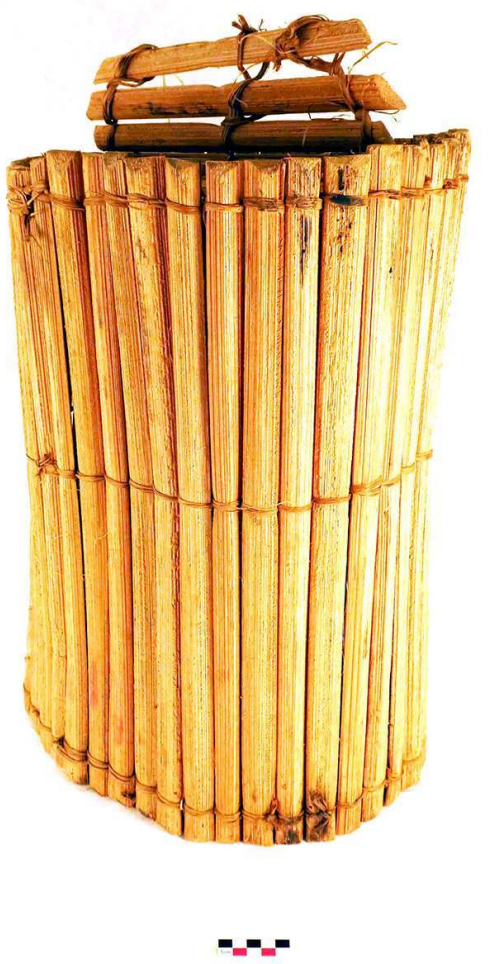

(C) P. Rameau 
- Lumbu, vili. Gabon. Socoma, Lagune Banio (Nyanga)

- En moelle de raphia. Pour conserver vivants les poisson-chats (cf. Sabinot 2008)

- 2005 ; collectrice Catherine Sabinot

- Dimensions : 46 x $26 ; 53$ x 36

- $\mathrm{N}^{\circ}$ d'inventaire : ETB-GA-CS-2005-002, ETB-GA-CS-2005-003

Figure 21 : Fabrication d'un vivier lumbu

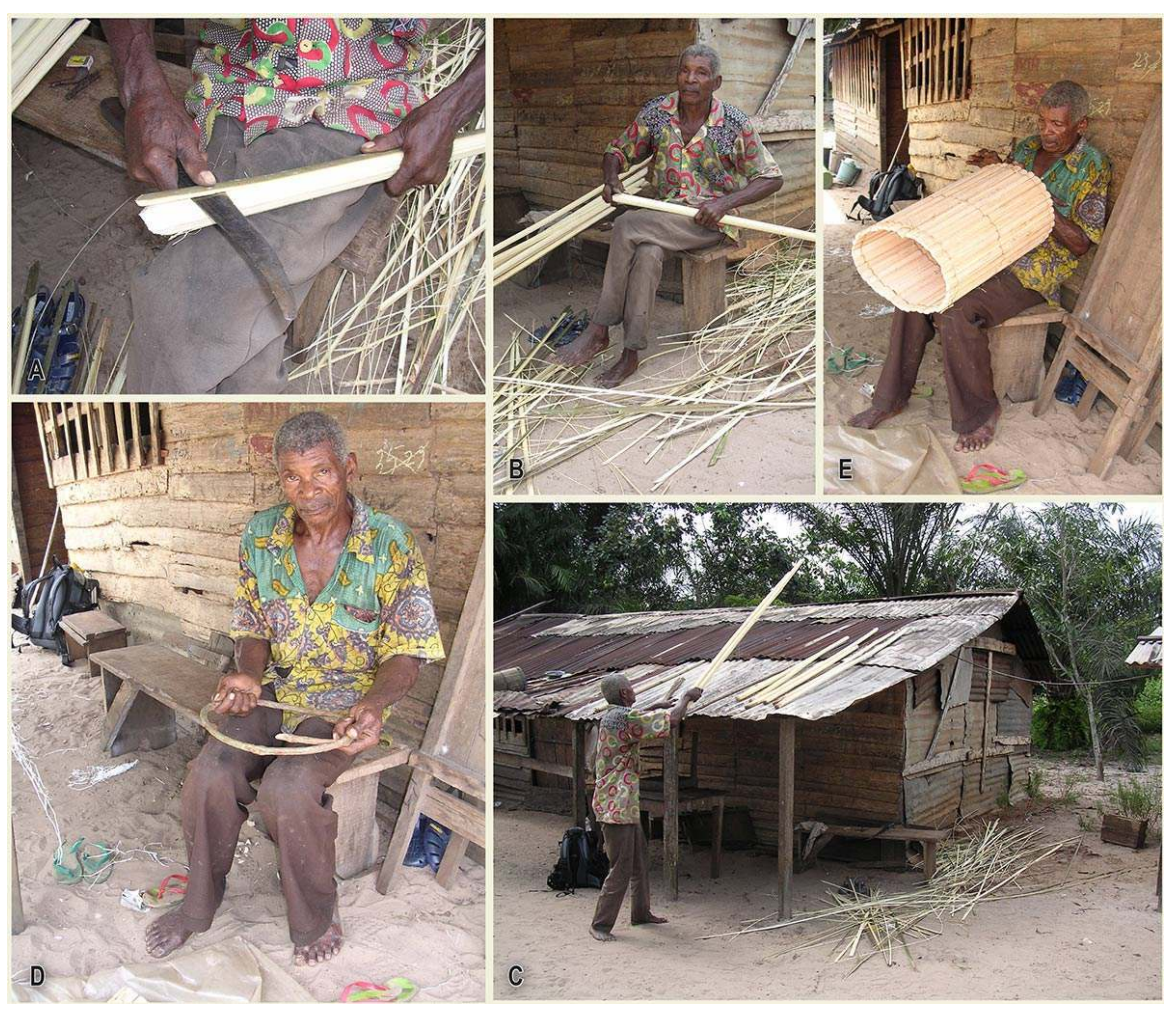

A: Écorçage des pétioles de Raphia - B: Préparation des cœurs de bambou - c: Les pétioles écorchés sont mis à sécher sur le toit- D: Préparation du cercle de rotin assurant la rigidité à l'intérieur du vivier - E: Assemblage du vivier (Socoma-Louando, lagune Banio, 2005)

(c) C. Sabinot

\section{Sans dispositif d'entrée}

C'est l'étroitesse du tube qui empêche le poisson entraîné par le courant de se retourner pour ressortir.

14 - « Nasse » tubulaire 
Figure 22 : Nasse tubulaire ngbaka ETB-CF-YM-2016-001

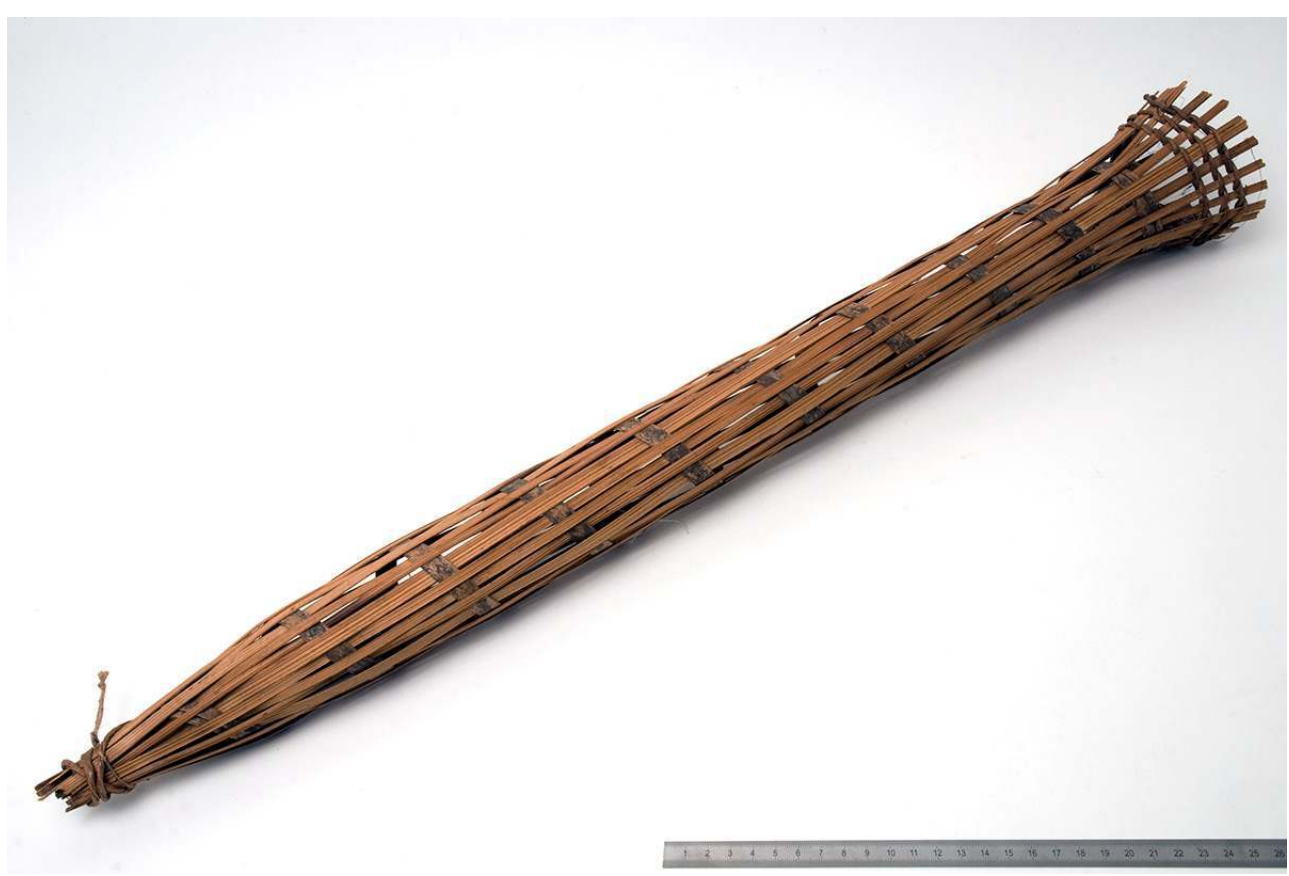

() J.C. DOMENECH/MNHN

- Ngbaka. RCA. Bobua-Bokanga (Lobaye)

- Ouverture simple, sans entonnoir. S'emploie dans un barrage, ouverture à contrecourant. Rotin

- 1970 ; collecteur Yves Moñino

- Dimensions : longueur $71 \mathrm{x}$ diamètre 6 , diamètre bouche 8,5

- $\mathrm{N}^{\circ}$ d'inventaire : ETB-CF-YM-2016-001 
Figure 23 : Barrage de pêche ngbaka avec diverses nasses. Mètè, RCA, 1980

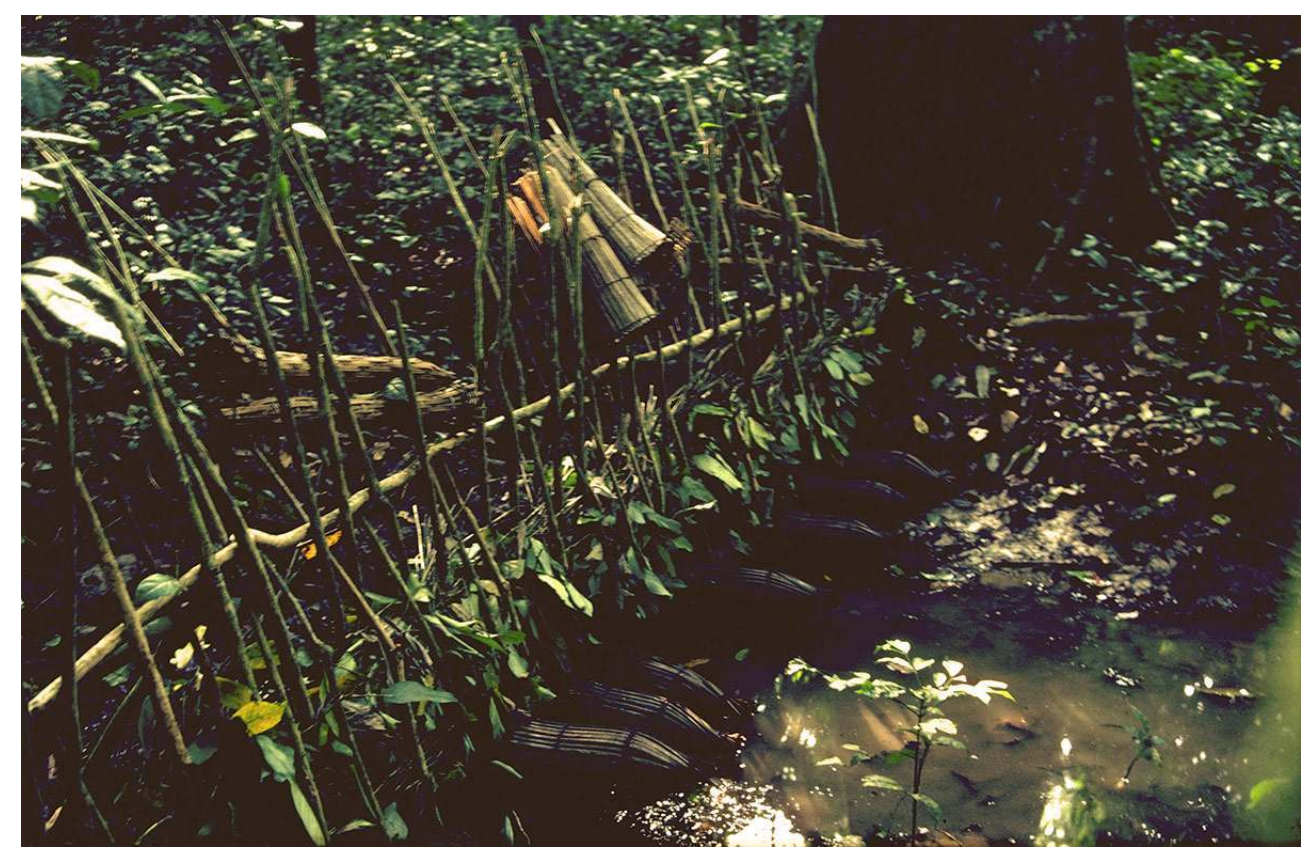

(c) S Bahuchet

\section{Déterrement-ramassage (classe XII)}

\section{Ramasser sur le sol, par assèchement d'une collection d'eau}

Le but de l'opération est la mise à sec d'une nappe d'eau. Une mare étant provoquée par des diguettes, l'eau ainsi retenue est vidée par écopage. En même temps que l'eau est projetée, un panier filtrant est maintenu dans son axe, afin d'intercepter petits poissons ou crevettes qui peuvent y être contenues. Cette technique associe donc deux ustensiles : une écope, et un panier filtrant, souvent manipulés par deux personnes.

\section{Les écopes}

\section{5 - Panier à écoper}


Figure 24 : Panier à écoper ngbaka ETB-CF-SB-2016-002

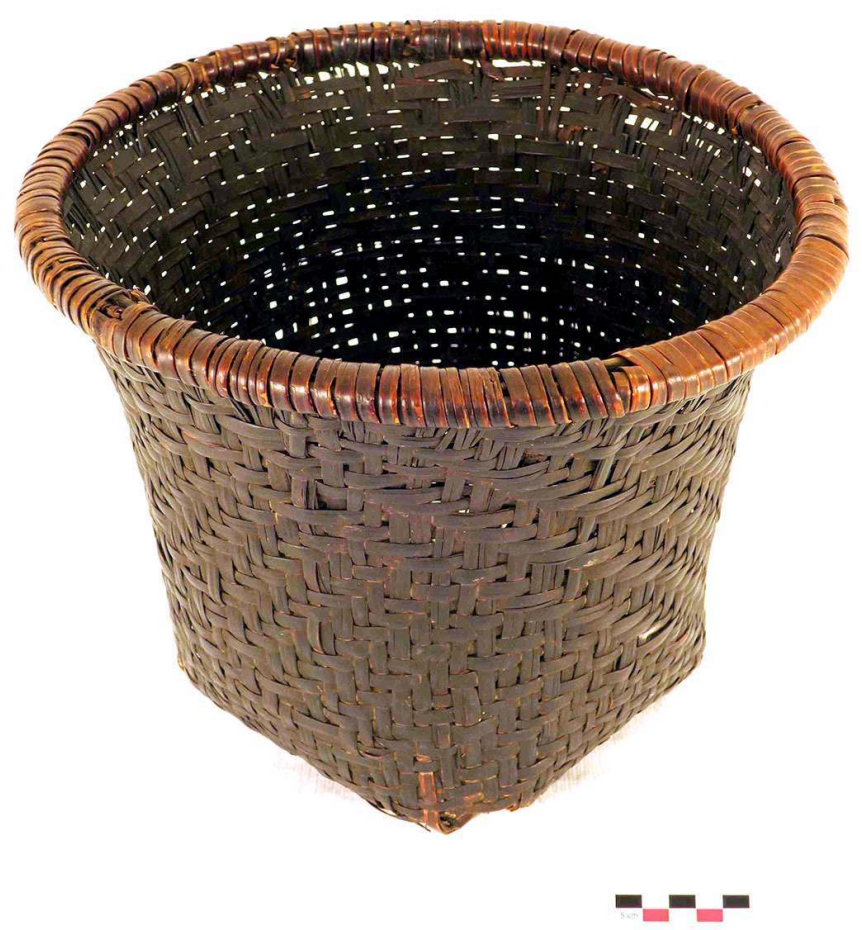

(C) P. Rameau

35 - Ngbaka, RCA, Mètè (Loko, Lobaye)

- Panier à tressage serré ; s'emploie avec le panier-filtre $n^{\circ} 22$ vers lequel on verse l'eau afin que les poissons y soient retenus. Lamelles de rotin

$-23,5 \times 31$

- 1980 ; collecteur Serge Bahuchet

- No d'inventaire : ETB-CF-SB-2016-002

16 - Cuvette de bois, plat à écoper 
Figure 25 : Cuvette à écoper ntumu ETB-CM-SB-2016-004

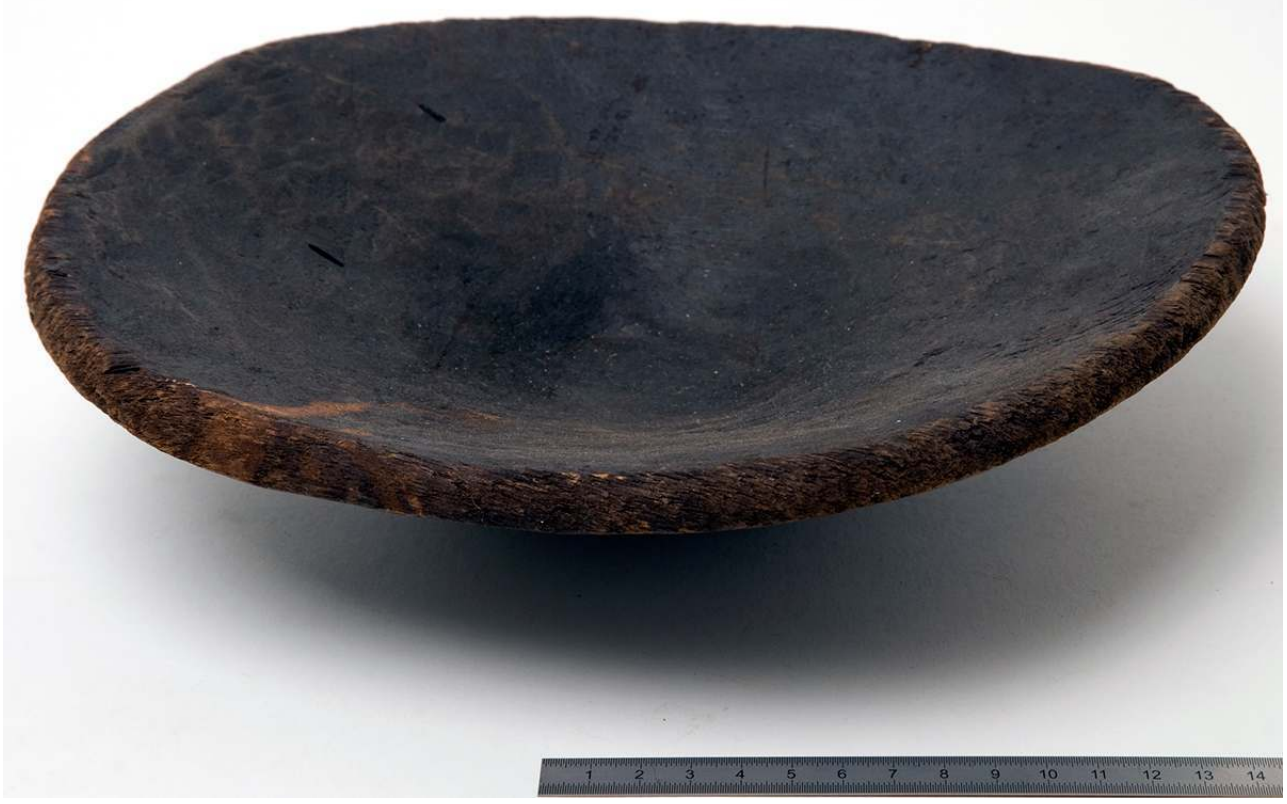

(c) J.C. Domenech/MNHN

- Ntumu, Cameroun, Nkong Meyos

- bois de contrefort de Fromager Ceiba pentandra (Bombacacée), durci et noirci au feu

- Décembre 1994 ; collecteur Serge Bahuchet

- Dimensions : 27 x 24 × 7

- $\mathrm{N}^{\circ}$ d'inventaire : ETB-CM-SB-2016-004

\section{Les filtres}

37 Bien qu'ils soient généralement manipulés en même temps qu'une écope, plusieurs de ces paniers servent également d'épuisette, tant dans les cours d'eau que dans les marres en cours d'assèchement.

\section{7 - Panier filtrant, simple}


Figure 26 : Panier filtrant bongo ETB-GA-MR-2007-001

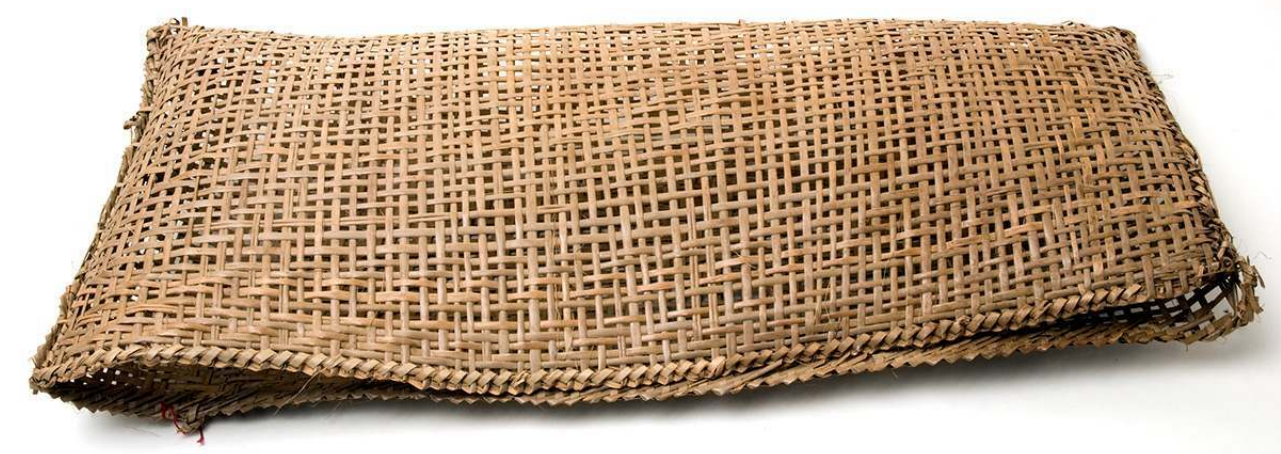

\section{(C) J.C. DOMENECH/MNHN}

- Bongo, Gabon, village Makoula (Ogooué-Lolo)

- Sert à filtrer l'eau dans les barrages sur les rivières, puis à transporter les petits poissons. C'est une petite natte en lamelles de Marantacées, pliée en deux et cousue sur les côtés

- Février 2007 ; collectrice Marine Robillard

- Dimensions : 67 x 31

- $\mathrm{N}^{\circ}$ d'inventaire : ETB-GA-MR-2007-001

Figure 27 : Départ à la pêche avec les paniers filtrant. Bongo, Mouyikou, mars 2015

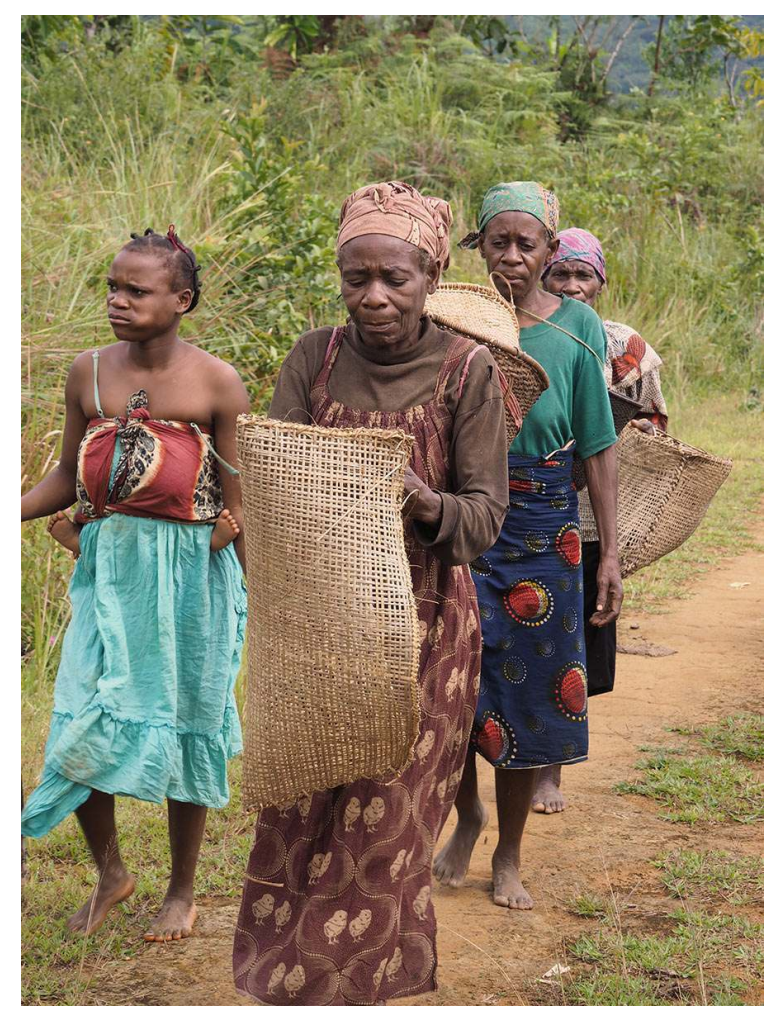

(C) S. Le Bomin 
Figure 28 : Panier filtrant bongo au cours d'une partie de pêche. Mouyikou, mars 2015

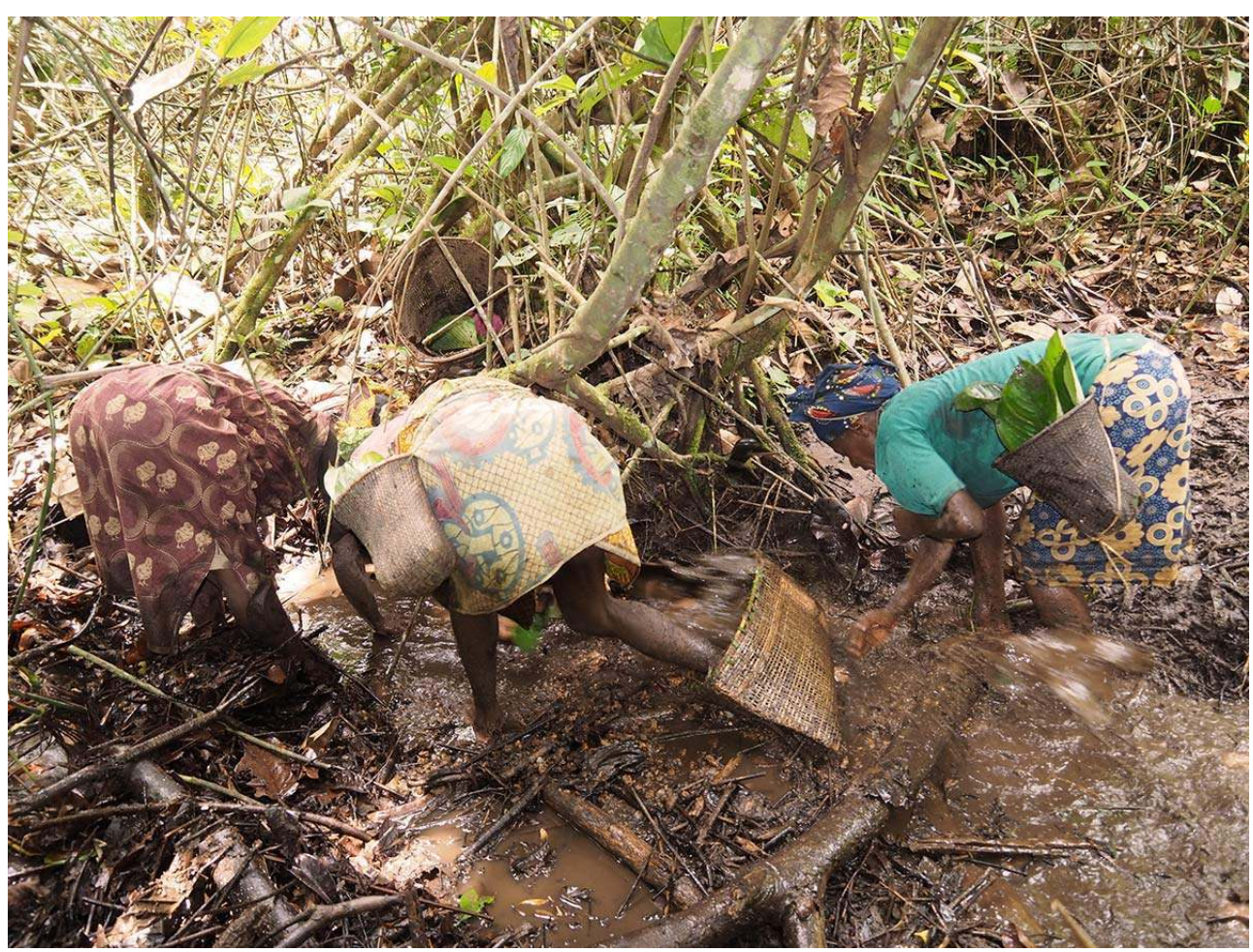

(C) S. Le Bomin

\section{8 - Panier filtrant, simple}

Figure 29 : Panier filtrant akele ETB-GA-SB/MR-2011-042

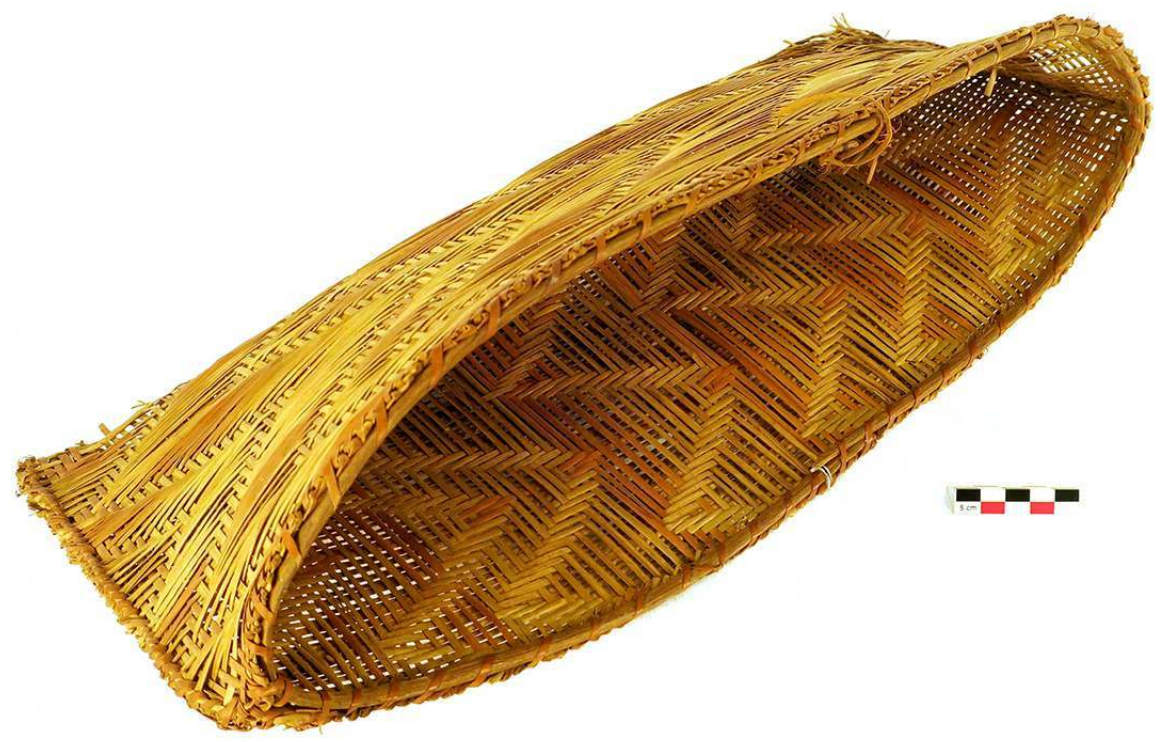

(C) P. Rameau 
- Akele, Gabon, village Dienga (Ngounié)

- Pour filtrer l'eau autour des barrages, et transporter les poissons. Lamelles de Marantacées. Armature de l'ouverture en rotin

- Avril 2011 ; collecteurs Serge Bahuchet, Marine Robillard et Sylvie Le Bomin

- Dimensions : 20 x 50,5 x 15

- $\mathrm{N}^{\circ}$ d'inventaire : ETB-GA-SB/MR-2011-042

\section{9 - Panier filtrant, simple}

Figure 30 : Panier filtre de l'Ivindo ETB-GA-SB-2016-001

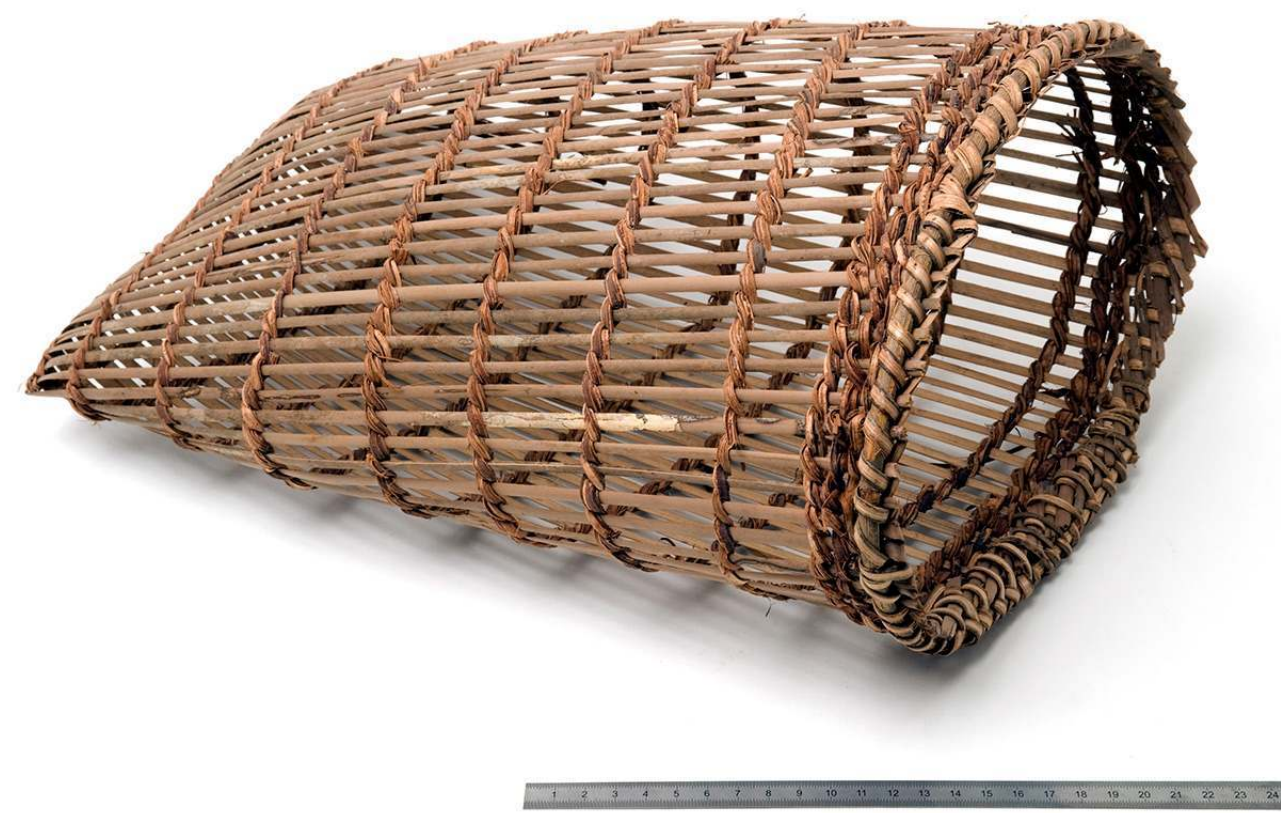

\section{(c) J.C. Domenech/MNHN}

- Probablement Kwele Gabon. Haut cours du fleuve Ivindo

- Panier fixé dans une barrière, sens opposé au courant; c'est la force de l'eau qui entraîne et bloque le poisson dans le panier. En lamelles de Marantacées

- Avril 1999 ; collecteur Serge Bahuchet

- Dimensions : 38 x 27 x 19

- $\mathrm{N}^{\circ}$ d'inventaire : ETB-GA-SB-2016-001

20 - Panier filtrant, avec poignée 
Figure 31 A et B : Panier filtrant bongo ETB-GA-SB/MR-2011-016

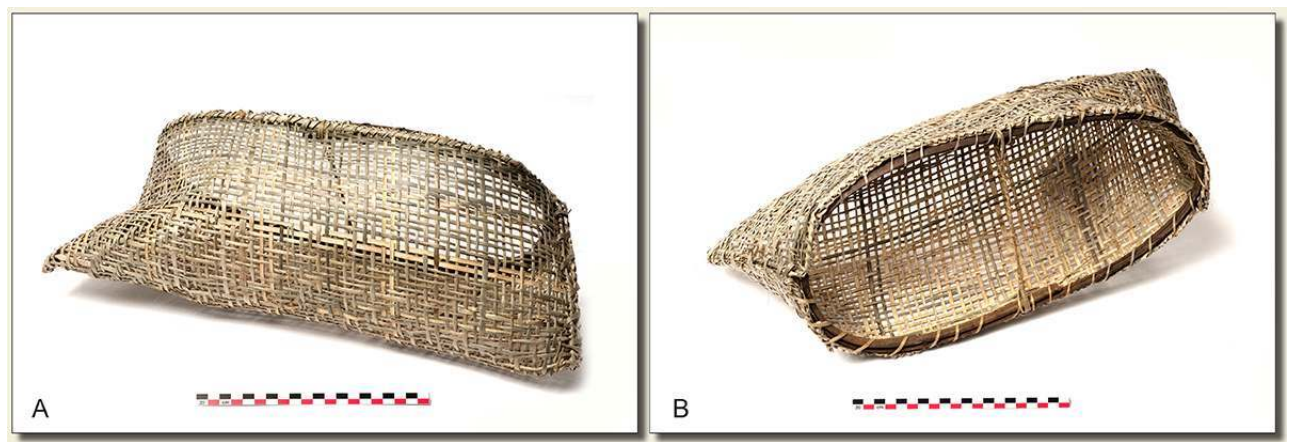

(C) J.C. Domenech/MNHN

41 - Bongo ; Gabon, Mouyikou, région de Mbigou

- Lamelles de Marantacées, et demi-tige de rotin pour la rigidité à la structure. Sert également de trouble

- Dimensions : 17 x 51,5 x 19,5

- 7 mai 2011 ; collecteurs Serge Bahuchet, Marine Robillard et Sylvie Le Bomin

- $\mathrm{N}^{\circ}$ d'inventaire : ETB-GA-SB/MR-2011-016

Figure 32 : Vanneries dans une case bongo, Mouyikou, Mais 2011

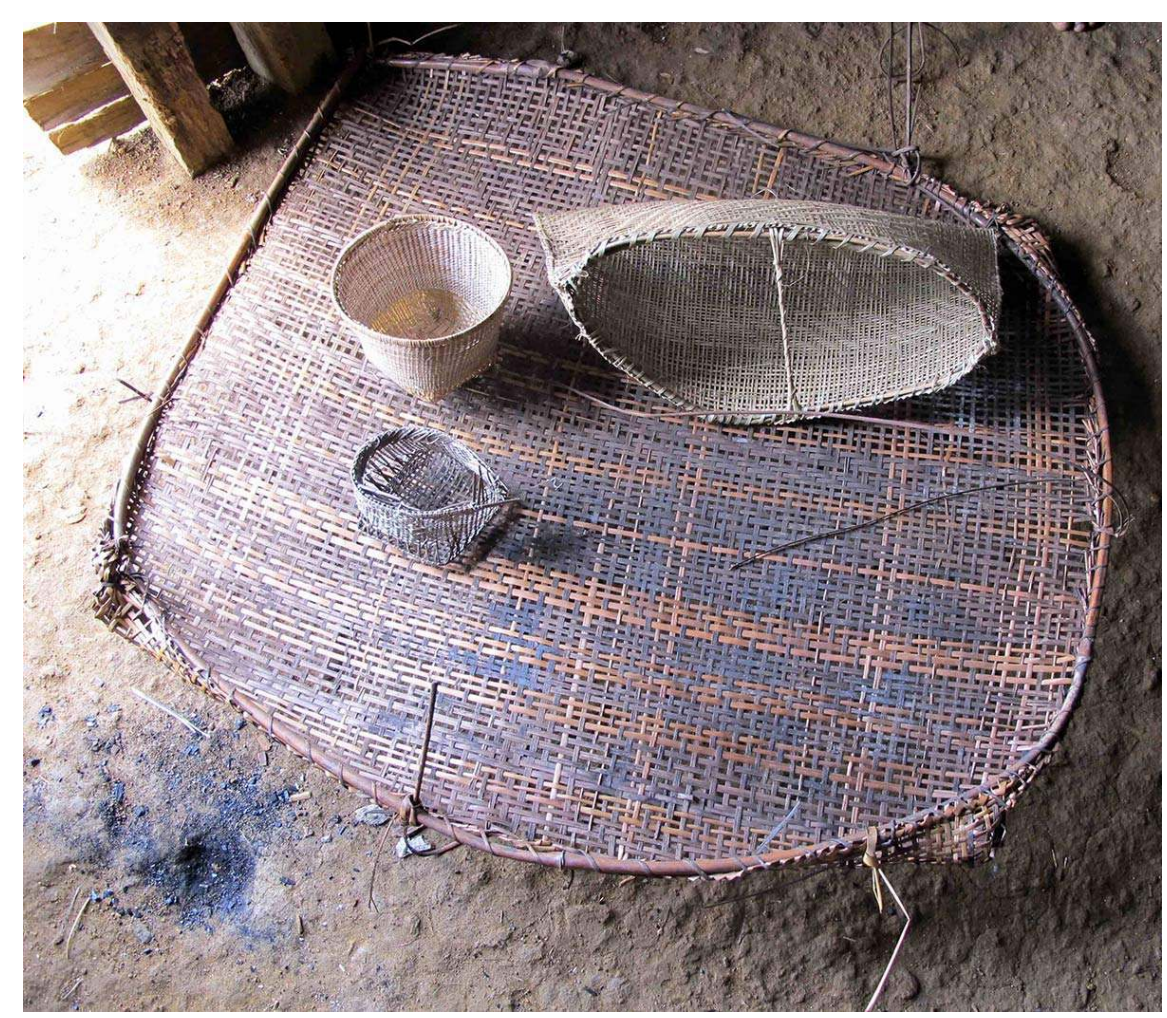

(C) S. Bahuchet

21 - Panier filtrant, avec poignée 
Figure 33 A et B : Panier filtrant bongo ETB-GA-SLB-2015-021
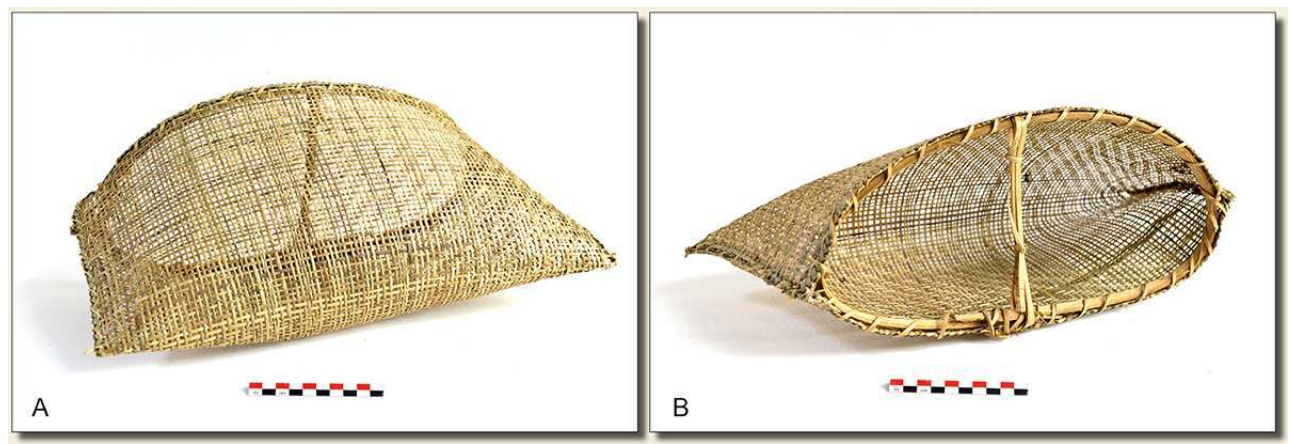

(c) J.C. Domenech/MNHN

42 - Bongo ; Gabon, Mouyikou, région de Mbigou

- Lamelles de Marantacées, et demi-tige de rotin pour la rigidité à la structure. Sert également de trouble

- Mars 2015 ; collectrice Sylvie Le Bomin

- Dimensions : 15 x 45 x 17

- $\mathrm{N}^{\circ}$ d'inventaire : ETB-GA-SLB-2015-021

Ce panier est exposé en permanence dans la Galerie de l'Homme

Figure 34 : Panier bongo au retour de la pêche. Mouyikou, mars 2015

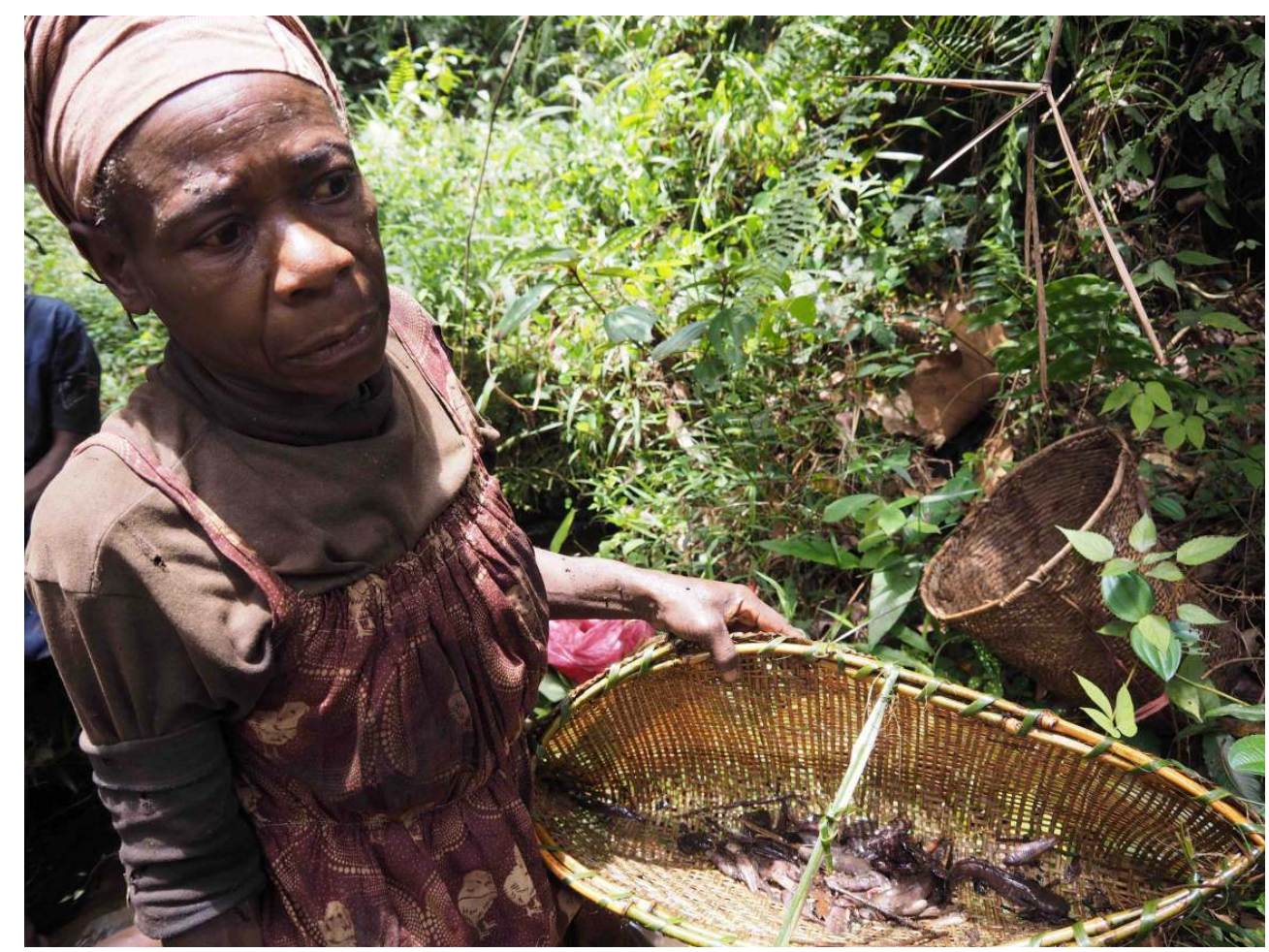

(c) S. Le Bomin

22 - Panier filtrant avec poignée 


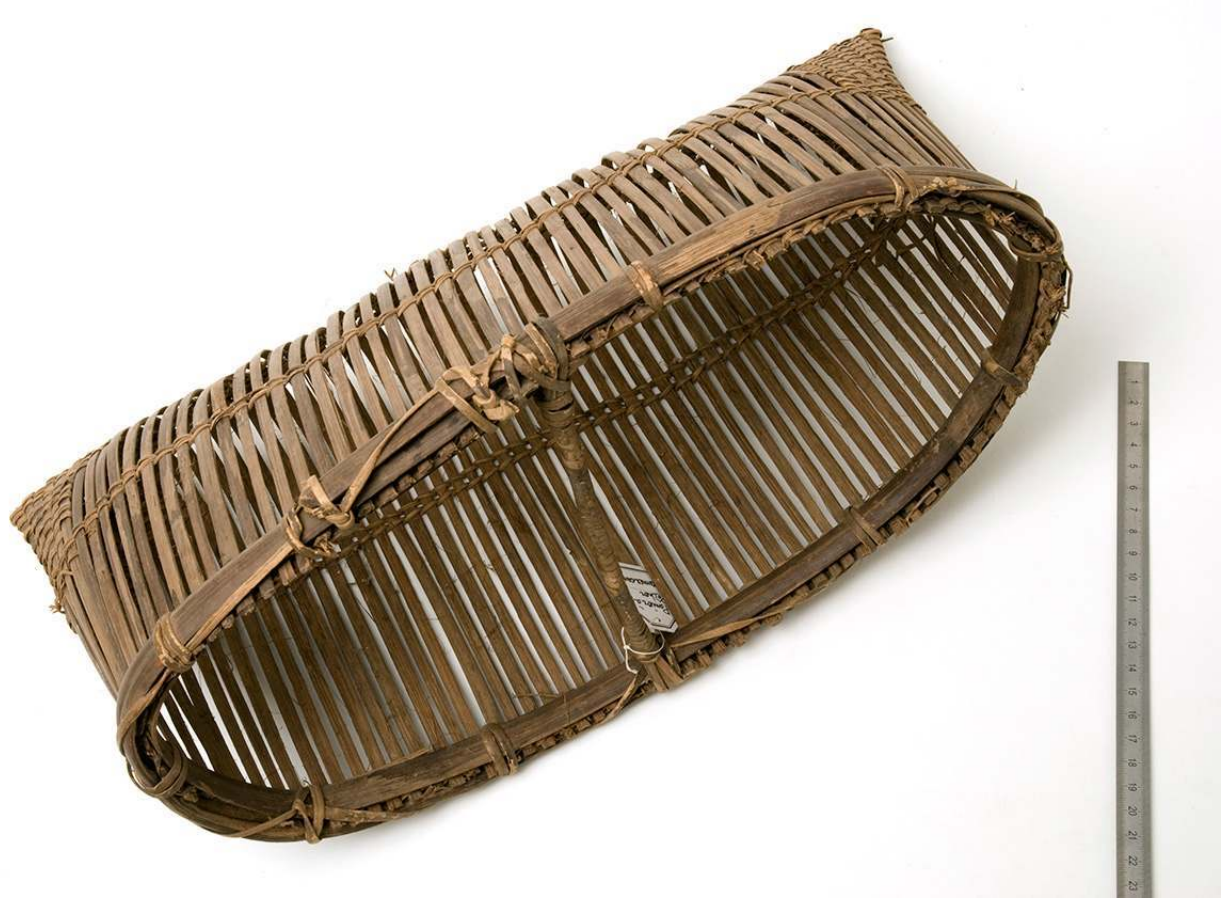

(c) J.C. Domenech/MNHN

44 - Ngbaka, RCA. Mète (Loko, Lobaye)

- Panier filtrant s'emploie avec le panier à écoper $n^{\circ} 15$. Lamelles de rotin. Sert également de trouble

- 1980 ; collecteur Serge Bahuchet

- Dimensions : 21,5 x 46 x 23

- $\mathrm{N}^{\circ}$ d'inventaire : ETB-CF-SB-2016-003

\section{Liste des objets par pays}

\section{Cameroun}

Ensemble de trois Hameçons: ETB-CM-SB-2016-003a, ETB-CM-SB-2016-003b, ETB-CMSB-2016-003c

Trouble, filet sur cadre : ETB-CM-SB-2016-002

Panier filtre : ETB-CM-SB-2016-001

Piège à crabe : ETB-CM-SB-2016-005

Écope en bois : ETB-CM-SB-2016-004

\section{Congo}

Deux Fers de harpons : ETB-CG-AE-2003-001, ETB-CG-AE-2003-002

Harpon emmanché : ETB-CG-AE-2003-003 


\section{Gabon}

Deux Fers de harpon : ETB-GA-AP-2012-001, ETB-GA-AP-2012-002

Nasse : ETB-GA-SB/MR-2011-043

Nasse : ETB-GA-SLB-2015-003

Nasse cylindrique : ETB-GA-CS-2005-001

Deux Paniers de ceinture : ETB-GA-SB/MR-2011-014, ETB-GA-SB/MR-2011-015

Panier filtre à poignée : ETB-GA-SB/MR-2011-016

Panier filtrant : ETB-GA-SLB-2015-021

Panier filtrant simple : ETB-GA-SB/MR-2011-042

Panier filtrant simple : ETB-GA-MR-2007-001

Panier filtrant simple : ETB-GA-SB-2016-001

Deux Viviers : ETB-GA-CS-2005-002, ETB-GA-CS-2005-003

\section{République centrafricaine (RCA)}

Grand panier filtre : ETB-CF-SB-2016-001

Nasse tubulaire : ETB-CF-YM-2016-001

Panier à écoper : ETB-CF-SB-2016-002

Panier filtrant avec poignée : ETB-CF-SB-2016-003

\section{République démocratique du Congo (RDC)}

Grande nasse : ETB-CD-VN-2013-001

\section{BIBLIOGRAPHIE}

Bahuchet S. 1992 - Esquisse de l'ethnoichtyologie des Yasa du Cameroun. Anthropos 87 : 511-520.

Bahuchet S., de Maret P., Grenand F., Grenand P. 2001 - Des Forêts et des Hommes : un regard sur les peuples des forêts tropicales. Ed. de l'Université de Bruxelles, APFT-ULB. 192 p.

Dounias E., Cogels S., Mvé Mbida S., Carrière S. 2016 - The safety net role of inland fishing in the subsistence strategy of multi-active forest dwellers in southern Cameroon. Revue d'ethnoécologie 10.

Froment A., Garine I. de, Binam Bikoy Ch., Loung J.F. (Ed.) 1996 - Bien manger et bien vivre. Anthropologie alimentaire et développement en Afrique intertropicale : du biologique au social. Paris, L'Harmattan-Orstom.

Hladik C.M., Bahuchet S., Garine I. de (Ed.) 1989 - Se nourrir en forêt équatoriale : anthropologie alimentaire des populations des régions forestières humides d'Afrique. UNESCO-MAB (Paris), 110 p. Leroi-Gourhan A. 1973 [1945] - Milieu et techniques. Albin Michel, Paris, 475 p. 
Monod T. 1973 - Contribution à l'établissement d'une classification fonctionnelle des engins de pêche. Bulletin du Muséum national d'Histoire naturelle, écologie générale 156 (12) : 205-231.

Nédélec C. 1982 - Définition et classification des catégories d'engins de pêche. FAO, Rome, 52 p.

Sabinot C. 2008 - Dynamique des savoirs et des savoir-faire dans un contexte pluriculturel. Étude comparative des activités littorales au Gabon. Paris, Thèse, MNHN, $388+82$ p.

Soengas López B. 2010 - La subsistance des Pygmées Bakoya à l'épreuve de l'agriculture : dynamique des savoirs ethnobotaniques et des pratiques. (Département de la Zadié, Ogooué-Ivindo, Gabon). Paris, Thèse, MNHN, $353 \mathrm{p}$.

\section{AUTEURS}

\section{SERGE BAHUCHET}

Professeur d'ethnobiologie du Muséum, laboratoire Eco-anthropologie et ethnobiologie (MNHNCNRS-Paris Diderot)

\section{PAULINE RAMEAU}

Chercheur associée, laboratoire Eco-anthropologie et ethnobiologie (MNHN-CNRS-Paris Diderot) 